\title{
Moving Beyond ERP Components: A Selective Review of Approaches to Integrate EEG and Behavior
}

\author{
David A. Bridwell ${ }^{1 *}$, James F. Cavanagh ${ }^{2}$, Anne G. E. Collins ${ }^{3,4}$, Michael D. Nunez ${ }^{5,6}$, \\ Ramesh Srinivasan ${ }^{5,6}$, Sebastian Stober ${ }^{7}$ and Vince D. Calhoun ${ }^{1,8}$ \\ ${ }^{1}$ The Mind Research Network, Albuquerque, NM, United States, ${ }^{2}$ Department of Psychology, University of New Mexico, \\ Albuquerque, NM, United States, ${ }^{3}$ Department of Psychology, University of California, Berkeley, Berkeley, CA, United States, \\ ${ }^{4}$ Helen Wills Neuroscience Institute, University of California, Berkeley, Berkeley, CA, United States, ${ }^{5}$ Department of Cognitive \\ Sciences, University of California, Irvine, Irvine, CA, United States, ${ }^{6}$ Department of Biomedical Engineering, University of \\ California, Irvine, Irvine, CA, United States, ${ }^{7}$ Research Focus Cognitive Sciences, University of Potsdam, Potsdam, Germany, \\ ${ }^{8}$ Department of ECE, University of New Mexico, Albuquerque, NM, United States
}

Relationships between neuroimaging measures and behavior provide important clues about brain function and cognition in healthy and clinical populations. While electroencephalography (EEG) provides a portable, low cost measure of brain dynamics, it has been somewhat underrepresented in the emerging field of model-based inference. We seek to address this gap in this article by highlighting the utility of linking EEG and behavior, with an emphasis on approaches for EEG analysis that move beyond focusing on peaks or "components" derived from averaging EEG responses across trials and subjects (generating the event-related potential, ERP). First, we review methods for deriving features from EEG in order to enhance the signal within single-trials. These methods include filtering based on user-defined features (i.e., frequency decomposition, time-frequency decomposition), filtering based on data-driven properties (i.e., blind source separation, BSS), and generating more abstract representations of data (e.g., using deep learning). We then review cognitive models which extract latent variables from experimental tasks, including the drift diffusion model (DDM) and reinforcement learning $(\mathrm{RL})$ approaches. Next, we discuss ways to access associations among these measures, including statistical models, data-driven joint models and cognitive joint modeling using hierarchical Bayesian models (HBMs). We think that these methodological tools are likely to contribute to theoretical advancements, and will help inform our understandings of brain dynamics that contribute to moment-to-moment cognitive function.

Keywords: EEG, ERP, blind source separation, partial least squares, canonical correlations analysis, representational similarity analysis, deep learning, hierarchical Bayesian model

Accepted: 06 March 2018 Published: 26 March 2018

Citation: Bridwell DA, Cavanagh JF, Collins AGE, Nunez MD,

Srinivasan $R$, Stober $S$ and Calhoun VD (2018) Moving Beyond ERP Components: A Selective Review of Approaches to Integrate EEG and Behavior.

Front. Hum. Neurosci. 12:106. doi: 10.3389/fnhum.2018.00106

\section{INTRODUCTION}

In the neural sciences, we are rarely afforded a one-to-one relationship between neural signals and phenotypic expression of a behavior or disease. It is increasingly common to use computational models to distill the latent features mediating brain-behavior relationships. Indeed, the field of computational psychiatry has emerged to formally address how such latent factors may inform clinically relevant expressions of disease (Montague et al., 2012; Huys et al., 2016). While this approach has leveraged neuroimaging to tie latent features to neural mechanisms, 
the field of human electrophysiology (electroencephalography, EEG) has been somewhat underrepresented by this approach. The purpose of this review article is to provide a theoretical and methodological manifest to advance the unique virtues of computationally-informed EEG. We first describe approaches to extract features from EEG, including time-frequency decomposition and machine learning approaches. Next we review the extraction of latent features from behavior derived from cognitive models. Finally, we highlight approaches to link these datasets post hoc with standard statistical tests, or to derive features jointly using data-driven joint decomposition, or cognitive joint modeling using hierarchical Bayesian models (HBMs).

EEG is commonly known as a useful neuroimaging measure due to its portability, affordability and high temporal resolution. In addition, EEG provides a direct measure of neural activity, since it reflects the aggregate synchronous synaptic activity of hundreds of thousands of radially oriented cortical pyramidal cells (Nunez and Srinivasan, 2006). There is a long history of literature demonstrating relationships between EEG oscillations and cognitive function (Klimesch, 1999; Jensen et al., 2007; Nyhus and Curran, 2010; Harmony, 2013), as well as the relationship between event-related potential (ERP) components and cognition and perception (Regan, 1989; Luck and Kappenman, 2012; Luck, 2014). However, researchers are beginning to focus on other features of EEG such as single trial transient events and non-sinusoidal fluctuations (Jones, 2016; Cole and Voytek, 2017). For example, waveform shapes may differ between different conditions (Cole et al., 2017), and amplitude modulations may be present within only a subset of peaks that follow the steady-state response (Bridwell et al., 2017).

ERP components are presumed to reflect latent computational operations (Luck, 2014), but they remain nebulously defined and are altered by experiment-specific (i.e., modality) and population level (i.e., age) factors, challenging their utility (Donchin et al., 1978). These issues are pointedly stated by Erol Basar in the book Brain Function and Oscillations in the following quote:

"Usually the averaged evoked response is described in terms of several arbitrarily defined components such as peak (wave) latencies and wave magnitudes. These arbitrarily defined components depend generally upon the location of the recording electrode, behavioral state or sleep stage of the subject under study, and upon the nature of the stimulating signal. Therefore, the interpretation of these arbitrarily defined components is very difficult..." (Basar, 1999)

It is widely observed but rarely formally acknowledged that event-related EEG activities reflect canonical neural operations that are predictably modulated within spatio-temporal windows. ERP components are only revealed by averaging across individual trials and subjects-these components (and their corresponding interpretations) may not hold at the individual subject or individual trial level (Rousselet and Pernet, 2011; Luck, 2014). For example, Gaspar et al. (2011) demonstrate that ERP responses to faces and noise are reliable across repeated measurements of the same subject, but that the individual subject averages differ among each other and with the group average. Thus, the emphasis on component peaks which appear following trial and subject averaging may detract from the ability to detect EEG features at the single trial or single subject level, and may limit the ability to relate those features with behavior.

Moreover, components are oftentimes defined by latent cognitive constructs (i.e., attention, working memory; Donchin and Coles, 1988; Folstein and Van Petten, 2007), which may not be effective characterizations of underlying neural computations. For example, it is not likely that different ERP components map onto the chapter titles within Psychology textbooks. In order to address these issues, we focus on deriving features from EEG which are not dependent upon the identification of canonical ERP component peaks (and their interpretations). For example, the processes which contribute to these stimulus and subject averaged responses may be decomposed based on their temporal frequency, and single trial data can be transformed to a lower dimensional representation using machine learning tools such as independent component analysis (ICA), representational similarity analysis (RSA), general linear modeling (GLM) and deep learning, as described in subsequent sections. We think that these methodological advancements are likely to contribute to theoretical advancements, and help to advance this field beyond a focus on ERP components.

Figure 1 provides an overview of the EEG and behavioral processing steps discussed within this review. The original EEG or behavioral signal represents the lowest level of abstraction, i.e., is closest to the original measurement, while various processing steps may be subsequently implemented to abstract from the original measurement using models that are justified mathematically, biologically, or both (in contrast to the data-driven focus within the EEG literature, behavioral measures are often directly linked with computational mechanism and cognitive theory via cognitive modeling; Turner et al., 2016). Within Figure 1, while the level of abstraction increases from top to bottom within the blocks for EEG and behavior, the level of sophistication of the approaches to link each dataset increases from top to bottom (as depicted within the center of the blocks in Figure 1). At the lowest level of sophistication, the EEG and behavioral features are derived independently and then linked post hoc, using standard statistical tests. At a higher level of sophistication, EEG and behavioral features are extracted independently and links are identified by decomposing the features within a common framework, i.e., joint data-driven decomposition such as joint-ICA (jICA) or multiset Canonical correlation analysis (CCA; i.e., "late fusion" approaches described within Dahne et al., 2015). Each level provides increasingly more information to the data fusion approach (Calhoun and Sui, 2016). Further, EEG features may contribute to the estimation of cognitive model parameters in parallel with direct measures of behavior (i.e., by specifying an explicit relationship between EEG features and behavioral features). HBMs have been implemented to accomplish linking in this manner, allowing EEG and behavioral features to jointly contribute to estimates of higher level cognitive processes (e.g., Frank et al., 2015; Nunez et al., 2017). 

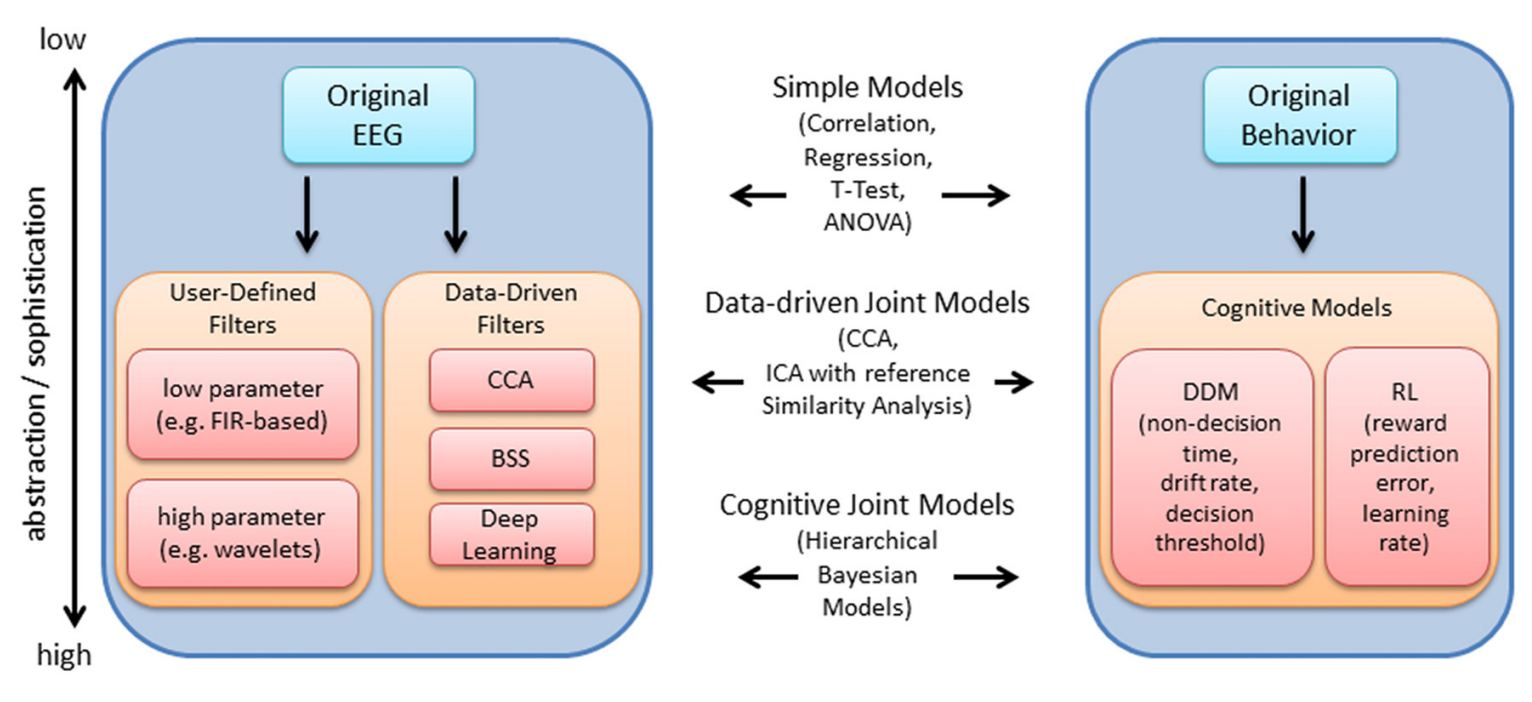

FIGURE 1 | Overview of electroencephalography (EEG) and behavioral processing steps reviewed, and approaches to integration. The original EEG data is depicted within the upper left. The arrow points to a series of processing steps which aim to extract features relevant to the experiment. These processing steps are generally comprised of user-defined (i.e., pre-selected) temporal filters with few or many parameters, or data-driven filters such as canonical correlation analysis (CCA), blind source separation (BSS) and deep learning. Within this article, we review time-frequency decomposition (with wavelets), various BSS approaches and deep learning approaches. These processing steps are useful for enhancing the signal at the single-trial level, which improves the ability to detect relationships between EEG and behavior. Using a similar depiction on the right, the original behavioral data (e.g., hit rate, false alarm rate, reaction time) may be used to derive latent measures of cognitive function (e.g., using drift diffusion models (DDM), or reinforcement learning (RL) models as examples). Measures at various levels of abstraction/sophistication within EEG and behavior may be combined using various approaches reviewed, including simple statistical models, data-driven joint models and cognitive joint models, as indicated in the middle of the plot.

By appropriately constructing models, these analyses should strive for understanding cognitive function at the single trial level within healthy and patient populations. Within the sections that follow, the EEG processing steps are described first, followed by the extraction of latent variables from direct measures of behavior. Then, simultaneous data-driven decomposition and joint approaches to integrate these measures are reviewed. Importantly, many of these methods can be implemented using publicly available toolboxes (see Table $\mathbf{1}$ ).

\section{OVERVIEW OF EXTRACTING FEATURES FROM EEG}

\section{Selecting User-Defined Features With Time-Frequency Filters}

Like a prism to sunlight, time-frequency filters decompose a time-series input into a dimension-expanded representation of frequency-specific temporal activities. In the context of EEG, filters like wavelets decompose the signal into biologically reasonable frequency bands (see Figure 2), and we focus on wavelets here as a representative example of a time-frequency filter.

Wavelet convolution operates by computing the similarity over time between the input signal and a template short-time frequency-specific oscillation (motivating the diminutive "let" after the word "wave" in "wavelet"). Morlet wavelets are constructed by multiplying a complex exponential carrier with a Gaussian window. A family of Morlet wavelets may include
50 frequency-specific sine waves (e.g., 1-50 Hz), each tapered (i.e., convolved) with a Gaussian function (Lachaux et al., 2002). This would reveal a dimension-expanded 50 -frequency by time matrix of activity from the input signal.

To understand this approach, it is important to know that convolution in the temporal domain is mathematically equivalent to multiplication in the frequency domain. This means that while wavelet convolution is a useful concept to understand how signal similarity underlies this transformation, the same output is usually computed by the much faster procedure of multiplying each of the Fourier coefficients for the input signal and the wavelet followed by reconstruction of the time-series via inverse Fourier transform. At its core, the wavelet procedure has some mathematical equivalence with other techniques for deriving time-frequency representations like the short time Fourier or band-passed Hilbert transform (Bendat and Piersol, 2010). However, these are oftentimes inexact replications, as parameters like filter width alter the output in subtle but meaningful ways. The pervasiveness of the Fourier transform across techniques offers an excellent way to double-check one's data processing for hidden bugs by comparing outputs derived via wavelet vs. filter-Hilbert techniques (Le Van Quyen et al., 2001; Yuan and Luo, 2012).

The dimension-expanded representation includes both the phase angle of the frequency-specific signal at each temporal moment (instantaneous phase) as well as the amplitude of this signal (usually squared to derive power). This decomposition is tremendously important for continued specification of the 
TABLE 1 | Select publicly available toolboxes for electroencephalography (EEG) analysis.

\begin{tabular}{|c|c|c|c|}
\hline Toolbox & Notable functions & Citation(s) & Link \\
\hline EEGLAB & BSS & Delorme and Makeig (2004) & http://sccn.ucsd.edu/eeglab \\
\hline EEGIFT & Group BSS & Calhoun et al. (2001) and Eichele et al. (2011) & http://mialab.mrn.org/software/eegift/ \\
\hline FIT & Joint ICA; CCA + Joint ICA; Parallel ICA & Calhoun et al. (2009) and Calhoun and Sui (2016) & http://mialab.mrn.org/software/fit \\
\hline EP Toolkit & PCA & Dien $(2010)$ & http://sourceforge.net/projects/erppcatoolkit/ \\
\hline LIMO EEG & Hierarchical Linear Modeling of EEG & Pernet et al. (2011) & http://github.com/LIMO-EEG-Toolbox/limo_eeg \\
\hline
\end{tabular}

BSS, blind source separation; ICA, independent component analysis; CCA, canonical correlation analysis; PCA, principle component analysis.

neural features that underlie cognitive operations. If multiple trials of an event are associated with consistent phase angles, the event is likely re-setting ongoing phase dynamics or instantiating a phase-dependent neural process (Makeig et al., 2002). This has strong implications for the nature of underlying neural networks that are created and dissolved over various time scales (and frequencies). Brain regions that are phase consistent are likely using phase dynamics to structure temporal windows for neurons to simultaneously fire and form a transient neural network (Fries, 2005). To clarify some unfortunately common terminology that appears in the field, wavelets do not reveal "oscillatory" activity, nor are oscillations necessary inputs for wavelet decomposition. Any time-series signal can be decomposed into a time-frequency representation; the repeating nature of the signal is irrelevant.

The process of wavelet convolution (or equivalently, Fourier coefficient multiplication) is conceptually and procedurally quite simple. However, many free parameters are critical for successful usage such as frequency range selection, taper dynamics, signal length and selection of an appropriate baseline to obviate the non-linear distribution of power between frequencies (i.e., the $1 / \mathrm{f}$ nature of EEG spectra). An interested scientist can find a full description of each of these issues as well as MATLAB code in the book Analyzing Neural Time Series Data (Cohen, 2014).

\section{Blind Source Separation}

Time-frequency decompositions (e.g., wavelets, discussed in the previous section) can emphasize signals that correspond to a distinct frequency band, removing artifacts that typically fall outside biologically plausible frequencies (e.g., $<1 \mathrm{~Hz}$ changes in skin impedance, high frequency muscle artifacts, and 50/60 Hz electrical line current). However, broadband artifacts and artifacts which overlap in frequency with biological oscillations are more problematic for this filtering approach. These scalp signals are superimposed with various sources of noise, including eye blinks, eye movement, muscle tension, heartbeat, AC electrical line current, and skin potentials. In addition, some sources of noise influence neural signals directly, e.g., when the content of visual stimuli changes with eye movements or blinks. Thus, various blind source separation (BSS) techniques have been applied to derive data-driven filters which disentangle these signal mixtures.

BSS approaches have been developed and implemented for matrix decomposition (Onton et al., 2006) or higher order ( $N \geq 3$ dimension) tensor decomposition (Cong et al., 2015) of EEG. These approaches provide an improved separation of EEG signal from EEG signal, or EEG signal from noise. Temporal ICA and principle component analysis (PCA) are two common EEG data reduction approaches with relative

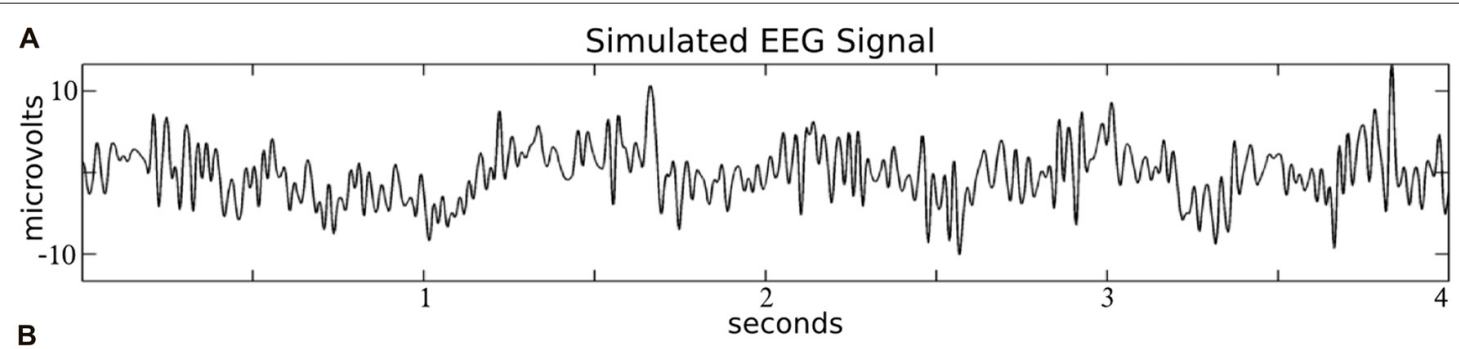

8

Wavelet Decomposed Signal

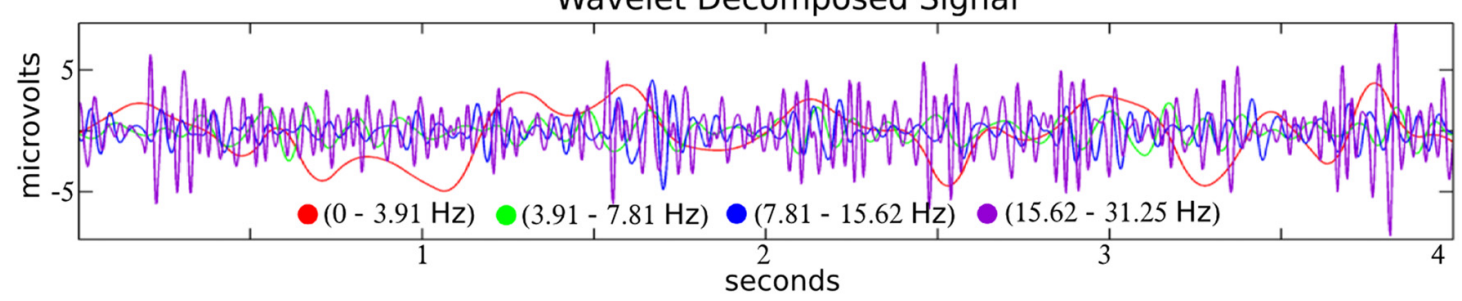

FIGURE 2 | Time-frequency decomposition of simulated EEG with wavelets. A simulated EEG signal (A; from the SIMEEG toolbox http://mialab.mrn.org/software/simeeg/index.html) was decomposed into time courses which correspond approximately to the delta (0-3.91 $\mathrm{Hz}$ ), theta $(3.91-7.81 \mathrm{~Hz})$, alpha $(7.81-15.62 \mathrm{~Hz})$ and beta $(15.62-31.25 \mathrm{~Hz})$, EEG frequencies (B). Wavelet coefficients were estimated using the discrete wavelet transform (DWT) implemented in MATLAB (http://www.mathworks.com) with the wavedec and wrcoef functions (biorthogonal spline mother wavelet; bior3.9; dyadic decomposition; 5 levels; Adapted from Bridwell et al., 2016). 
advantages and disadvantages that depend on the degree in which the data aligns with the methods assumptions (Bugli and Lambert, 2007; Dien et al., 2007). PCA derives a set of latent variables which are uncorrelated, while temporal ICA decomposes the data into a linear mixture of temporally independent sources and their associated loadings or mixing matrix (i.e., scalp topography; Makeig et al., 1997; Hyvarinen et al., 2001; Stone, 2004). Temporal ICA has been particularly popular since assumptions of the approach are consistent with the linear mixture of independent cortical electrical potentials that occurs across the scalp due to volume conduction. And temporal ICA is particularly robust as a pre-processing step in EEG artifact removal, since eye blinks and eye movement artifacts are independent and non-Gaussian sources which contaminate the EEG. These artifactual sources may be removed before converting the data back to the original channel space (Jung et al., 2000; Makeig et al., 2004; Onton et al., 2006).

Temporal ICA and PCA have demonstrated utility in decomposing the multiplexed ERP response into distinct ERP peaks (for temporal ICA, see Onton et al., 2006; Bridwell et al., 2014, 2015, for PCA see Bernat et al., 2005), which likely leads to a more accurate estimate of the amplitudes of distinct ERP components than the original superimposed response. However, these models suffer from similar caveats as many of the modeling approaches discussed-they require extensive user attention and parameter selection (e.g., PCA components may differ depending on how many are selected for rotation, the type of rotation and how the data are conditioned; Kayser and Tenke, 2003; Dien et al., 2005).

In general, artifacts are readily separated from EEG sources with temporal ICA due to their non-Gaussian distribution (Hyvärinen et al., 2010). However, temporal ICA seems ill equipped at separating non-artifact sources, motivating the use of alternative BSS approaches for decomposing different EEG oscillations (e.g., EEG collected in the absence of an explicit task), including algorithms designed to decompose EEG oscillations explicitly and those that decompose real or complex valued EEG spectra (Anemüller et al., 2003; Bernat et al., 2005; Hyvärinen et al., 2010; Porcaro et al., 2010; Nikulin et al., 2011; Shou et al., 2012; Bridwell et al., 2016). These approaches are well suited for decomposing the broad EEG spectra into functionally distinct frequency bands, including separating the alpha band $(8-12 \mathrm{~Hz})$ into its low and high frequency sub-bands (Niedermeyer, 1997; Nunez et al., 2001; for an example, see Bridwell et al., 2013).

Various extensions to BSS have been developed to address the issue of determining which BSS sources correspond across subjects, including approaches which decompose the aggregate group EEG data (Kovacevic and McIntosh, 2007; Congedo et al., 2010; Eichele et al., 2011; Cong et al., 2013; Lio and Boulinguez, 2013; Ponomarev et al., 2014; Ramkumar et al., 2014; Huster et al., 2015; Huster and Raud, 2018; Labounek et al., 2018). Within temporal Group ICA implemented in EEGIFT ${ }^{1}$, sources are estimated at the aggregate group level and individual subject sources are recovered by back-reconstruction (Calhoun et al., 2001; Calhoun and Adali, 2012). This approach effectively

\footnotetext{
${ }^{1}$ http://mialab.mrn.org/software/eegift/
}

"filters" the individual subject data by the source activity that is robust at the group level. However, the emphasis on the aggregate group dataset introduces issues in generating reliable decompositions in cases where there are topographic differences across subjects (Huster et al., 2015). Nevertheless, Group ICA is particularly advantageous in cases where individual trials are analyzed, as demonstrated by Bridwell et al. (2014), where single trial ERP amplitudes were correlated with single trial measures of prediction error or surprise. Within further studies, these single trial amplitudes would provide useful input measures to simple and joint models which integrate EEG and behavior (e.g., as discussed in the sections on Single Trial Regression and HBMs).

\section{Deep Learning}

Deep learning is a subfield of machine learning that during the last decade has gained substantial popularity by breaking benchmark records in various domains (such as computer vision, automatic speech recognition, natural language processing and bioinformatics). The term deep learning was coined as a reference to deep artificial neural networks (ANNs) with more than one hidden (i.e., non-input or -output) layer of neurons. Most notably, these techniques are able to learn complex non-linear functions that transform raw signals into feature representations or derive probability distributions over latent variables. The latter can be achieved through probabilistic graphical models like restricted Boltzmann machines (RBMs) or deep belief nets (DBNs). Popular approaches for the former are convolutional and recurrent neural networks-CNNs and RNNs respectively (for a review in the context of neuroimaging, see Plis et al., 2014). In a convolutional layer, the input is convolved with trainable filters as illustrated in Figure 3, whereas recurrent connections feed the layer output back into earlier layers as additional input. All these techniques support unsupervised

\begin{tabular}{|c|c|c|c|c|c|c|c|c|c|}
\hline \multicolumn{5}{|c|}{$\begin{array}{c}\text { convolution } \\
\text { with } 3 \times 3 \text { kernel }\end{array}$} & \multicolumn{5}{|c|}{$\begin{array}{l}2 \times 2 \text { max-pooling } \\
\text { with stride } 1 \times 1\end{array}$} \\
\hline 11 & 0 & 1 & 0 & 0 & \multirow[b]{2}{*}{4} & \multirow[b]{2}{*}{3} & \multirow[b]{2}{*}{4} & & \\
\hline 0 & 1 & 9 & 1 & 0 & & & & 4 & 4 \\
\hline 10 & O & 1 & 1 & 1 & 2 & & 3 & \multirow{2}{*}{4} & 4 \\
\hline 0 & 0 & 1 & 1 & 0 & 2 & 3 & 4 & \multirow{3}{*}{\multicolumn{2}{|c|}{$\begin{array}{l}\text { pooled } \\
\text { feature }\end{array}$}} \\
\hline 0 & 1 & 1 & 0 & 0 & \multirow{2}{*}{\multicolumn{3}{|c|}{$\begin{array}{l}\text { convolved } \\
\text { feature }\end{array}$}} & & \\
\hline \multicolumn{5}{|c|}{$\begin{array}{l}\text { 2D input } \\
\text { (1 channel) }\end{array}$} & & & & & \\
\hline \multicolumn{10}{|c|}{$\begin{array}{l}\text { FIGURE } 3 \text { | Illustration of a convolution operation with a single } 2 \mathrm{~d} 3 \times 3 \text { kernel } \\
\text { (orange) applied to a } 2 \mathrm{~d} 5 \times 5 \text { input. In a convolutional layer, the input is } \\
\text { convolved with trainable filters. Generally, input and kernels may have different } \\
\text { shapes and span multiple channels (not shown here). Further, multiple kernels } \\
\text { can be applied in parallel within the same convolutional layer. The convolution } \\
\text { is commonly followed by an element-wise application of a non-linear } \\
\text { transformation (not shown) and optionally a pooling step (blue) that } \\
\text { aggregates neighboring output values. }\end{array}$} \\
\hline
\end{tabular}


training for signal modeling and learning high-level patterns as well as supervised training for discriminative tasks such as classifying EEG based on the recorded trial type or upon collected behavioral measures. Importantly, the approach can incorporate the entire spatial and temporal structure, and the outputs of the kernel may be visualized as topographic feature maps (Figure 4; Bashivan et al., 2016).

A common approach for unsupervised training of ANNs which goes back to the 1980s is the auto-encoder (Bourlard and Kamp, 1988). Such a network consists of an encoder and a decoder. The encoder transforms input data into an internal representation whereas the decoder computes a reconstruction of the input from this representation. The whole network is trained by minimizing the reconstruction error for some training dataset. In order to make this task non-trivial and force the auto-encoder to learn meaningful features that represent the data well, a representational bottleneck is commonly required-for instance, limiting the number of neurons in the inner-most layer (structural bottleneck), adding noise to inputs or activations, or sparsity regularizations. Auto-encoders can be stacked to incrementally learn higher-level features.

With respect to supervised learning approaches, a neural network pre-training technique for learning discriminative features from stimulus-driven EEG signals was described by Stober (2017). Demanding that encodings of two trials from the same class are more similar to each other than to encoded trials from other classes, a respective encoder pipeline was trained that improved the signal significantly even across subjects. This tuple-based training approach is especially suitable for small datasets and measures with a low signal-to-noise ratio (SNR), such as EEG.

In applying deep learning to EEG datasets, there are a few important considerations. Investigators should be careful in instances where unrealistically high accuracy is observed, as this is usually an indicator of a flawed experiment. For instance, the way the data was recorded and split into subsets for training and evaluation could have introduced artifacts that were exploited by the classifier. This so-called "Horse" phenomenon has, for instance, been described by Sturm (2014). Another potential cause for flawed results are software bugs such as mistakes in indexing trials or accidentally duplicating trials that result in overlapping training and evaluation data.

\section{OVERVIEW OF ESTIMATING LATENT VARIABLES FROM BEHAVIOR}

The previously discussed EEG processing steps derive useful features which may be subsequently related to behavior. Within this section, we review approaches to integrate latent cognitive variables for linking with EEG within subsequent steps, which differs from the typical focus on observable, experimenterdefined objective variables. For example, categorical variables (e.g., stay vs. switch trials) are used to pool trials into groups across which the neural signal is then compared (Collins et al., 2014), or continuous variables (e.g., the amount of conflict) can be used for single trial analysis of the EEG signal (Cohen and Cavanagh, 2011). This categorical approach can be improved by implementing cognitive modeling (i.e., computational modeling of behavior) techniques to estimate latent variables on a trial by trial basis, and subsequently relating these measures with EEG.

Computational models of behavior usually propose a mechanistic or algorithmic description of the computations that may be happening in the brain to support behavior. These models usually have parameters (e.g., drift rate or learning rate, see below for details), that quantitatively modulate the computations made by the model. Model fitting techniques allow us to infer the parameters that are most likely to give rise to the observed behavior. Then, given a set of parameters for a model, it is also possible to obtain the latent variables that are part of the models' computations, and thus that putatively are the underlying variables needed to account for the observed behavior.

Thus, cognitive modeling may provide two types of benefits to relate behavior and EEG signal. First, fitting computational models to behavioral data allows researchers to extract model parameters that are putatively related to mechanisms underlying behavior; these parameters may then capture variability (between
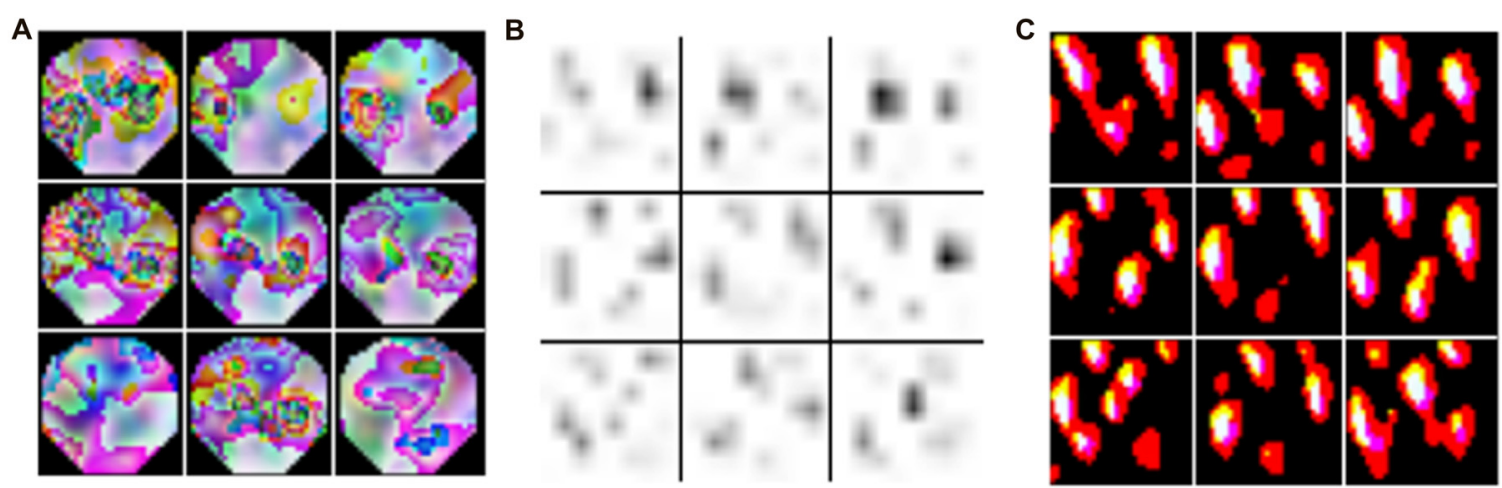

FIGURE 4 | Topographic features derived from kernels in recurrent-convolutional neural networks. The input EEG with the highest activation across the training set is indicated in (A). Feature maps were derived from the kernel (\#122 in the 3rd stack output) and plotted in (B), and back-projected topographies were computed using deconvnet in (C). The figure is modified from Bashivan et al. (2016) and reproduced with permission. 
conditions or individuals) better than raw behavior would. Second, model fitting also allows researchers to extract latent variables that putatively reflect the computations supporting behavior. These variables may then be better candidates to reflect the trial-by-trial neural signal. In the next paragraphs, we give an overview of the computational modeling process with these two target benefits, and focus on two key examples: drift diffusion modeling (DDM) for perceptual decision making (Ratcliff, 1978; Ratcliff and McKoon, 2008), and reinforcement learning (RL) modeling (Sutton and Barto, 1998).

The DDM is a special case of a sequential sampling model (SSM) we focus on here. The DDM is a used to account simultaneously for accuracy and reaction time observations in binary perceptual decision tasks, such as the random dots motion task (e.g., Roitman and Shadlen, 2002). Specifically, the DDM formalizes decision as a noisy accumulation of evidence to one of two bounds; it assumes that once the decision variable reaches the bound, the corresponding choice is made. The DDM is usually parameterized with three parameters: non-decision time, drift rate and threshold. The non-decision time reflects a fixed period of time during which no information is accumulated; mechanistically, it may include both initial perception latency and motor command latency after the decision is made. The drift rate reflects the rate at which information is accumulated, or the strength of each new piece of evidence. The threshold indicates the level the evidence should reach prior to a decision being taken. Other parameters are sometimes included in the DDM to better capture behavior; for example, a bias term may be needed to capture participants' tendency to select one option more than another (see Figure 5A).

Simulations of the DDM can show how changing the parameters modifies performance, and reaction times for different conditions (e.g., correct vs. incorrect trials; Ratcliff and McKoon, 2008). For example, with a higher threshold, more evidence accumulation is required before reaching a decision, which is less likely to happen due to noise; thus, the observed behavior is more conservative: fewer errors with longer reaction times. Slower drift rates produce a qualitatively similar pattern, but quantitatively different. We thus need a quantitative method to disentangle the role of different parameters in the observed behavior of participants. The model fitting procedure computes a measure of fit, the ability of the model to predict the distributions of choices and reaction times across the two conditions for a given set of parameters; it then attempts to maximize this fit measure to find the set of parameters that best explain the behavior. Fitting can be done separately for different subjects or conditions, and fit parameters can then be related to neural signal-for example, frontal theta power and DDM threshold (Cavanagh et al., 2011).

Model fitting can also provide inferred trial-by-trial latent variables on which decisions rely. For example, in a learning task where participants learn to select the best out of two options using probabilistic feedback (Frank et al., 2004), the inferred learned value of each option at each time point is a continuous variable of interest that is not directly observable. RL models assume that participants track an estimate $V_{t}$ of the value of different options at each time $t$, and that they update this estimate when they obtain a reward $r_{t}$ by incrementing it by a proportion of the reward prediction error (RPE), the difference between the observed reward and the predicted reward: $r p e=r_{t}-V_{t}$. By how much the estimate is incremented is controlled by the learning rate parameter $\alpha$, such that the new estimate at time $t+1$ is $V_{t+1}=V_{t}+\alpha r p e$. The choice between two options depends on the estimated values, and can be controlled by a noise parameter (e.g., the softmax inverse temperature $\beta$; see Figure 5B).

The best parameters for a participant can be obtained by a similar model-fitting procedure to the one described for the DDM. Once the parameters are inferred, researchers can deduce a sequence of latent variables defined by the computational model, the fit parameters and participants' choice and reward history. For example, in a probabilistic learning task, we can
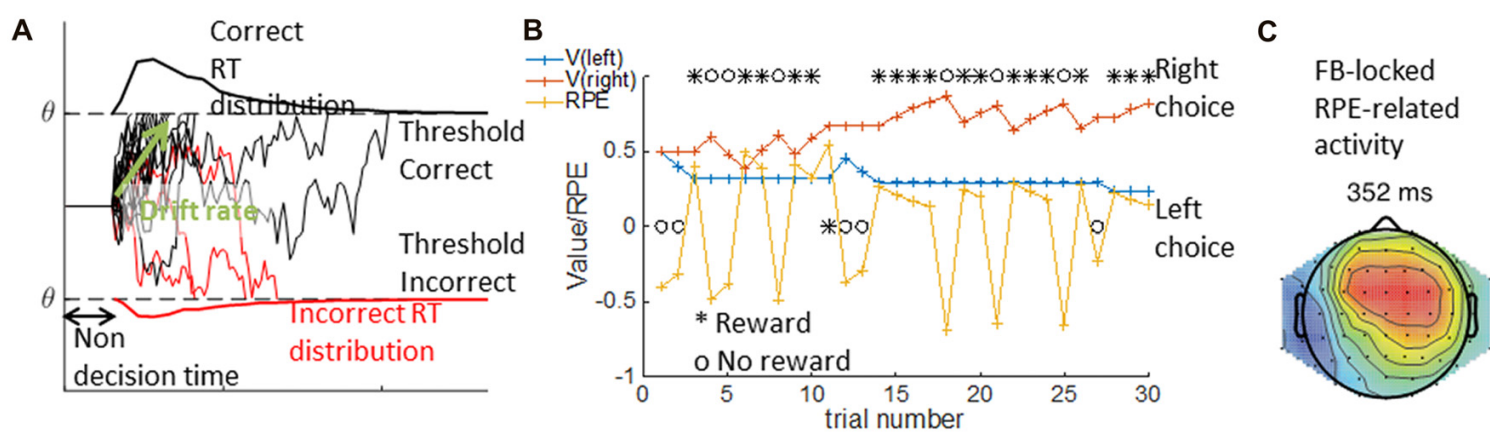

FIGURE 5 | DDM and RL Model. In the DDM in (A), single traces show multiple examples of simulated noisy accumulation of evidence to correct (black) or incorrect (red) decisions. The resulting distribution of reaction times are plotted for correct (top) and incorrect trials (bottom). The DDM relies on three main parameters - the non decision time, the threshold $q$ indicating the bounds to which evidence is accumulated, and the drift rate indicating the rate of evidence accumulation. In the RLM depicted in (B), an example of a sequence of 30 learning trials is given where the left choice is rewarded with probability $p=0.2$, and the right with probability $p=0.8$. Given a choice and reward history (black), the computational model provides the inferred underlying changes in expected value for each option ( $\mathrm{V}$, red and blue traces), and the inferred reward prediction error (RPE, yellow). The latent variables from modeling can be used to analyze trial by trial voltage. Within (C) activity over mid-frontal electrodes is correlated with RPEs from correct trials (modified from Collins and Frank, 2016). 
extract the sequence of expected values and RPEs that best explain participants' choices. These latent variables can then be linked to the neural signal on a trial by trial basis (e.g., Collins and Frank, 2016, and Figure 5C), thus giving insight into the computational mechanisms underlying behavior.

While using computational models for analyzing behavior and linking it to the neural signal can be extremely helpful, it also has many potential pitfalls that should be carefully kept in mind, and a number of methods can mitigate them (Nassar and Frank, 2016). The most important step is the validation of the model. It is impossible to be sure that a given model is a good mechanism for a cognitive process of interest, however, it is important to ensure that it is a reasonable candidate. A relative measure of a model's goodness includes comparing its fit to other candidate models. Quantitative comparisons should take into account model complexity, as more complex models are more likely to overfit the data. A more absolute measure of a model's goodness of fit requires posterior predictive checking: it is important to simulate a model that would result from the best-fit parameters and ensure that the pattern of simulated behavior is a qualitative match to participant performance.

\section{DATA FUSION: LINKING EEG/ERPs AND BEHAVIOR IN AN EXPLORATORY/DATA-DRIVEN MANNER}

In the sections that follow, we highlight approaches to link EEG data and behavioral data without imposing cognitive modeling constraints on their relationships. Single trial regression identifies associations between the two measures based upon their linear relationships, while the data-driven approaches (i.e., data-driven joint models) link EEG and behavior based upon the particular assumptions of the BSS model. These approaches do not incorporate an explicit link to cognitive parameters as the joint cognitive models discussed within the subsequent section (see "Linking EEG/ERP and Behavior With Latent Variable Estimation (Hierarchical Bayesian Models)" section), but nevertheless hold considerable utility in revealing the relationship between EEG responses and behavior.

\section{Single Trial Regression}

Trial-by-trial correlations between neural signals and behavior offer the most stringent correlational test of brain-behavior relations. Yet, it is arguably more common to see brain-behavior correlations computed between, rather than within individuals. While this can account for important individual differences, it does not offer a mechanistic test of how the brain facilitates behavior.

As an example, consider the question about how self-generated error signals motivate behavioral correction. The Error-Related Negativity (ERN) is a robust EEG marker of motor errors of commission (Falkenstein et al., 1991; Gehring et al., 1993), and early reports showed that it predicted slower response times following the error: an important manifest indicator that may indicate the application of cognitive control (Laming, 1979; Gehring et al., 1993). Subsequent studies contributed inconsistent evidence for a predictive relationship between MCC control signals and behavioral adjustments (see Weinberg et al., 2012). The ambiguity, however, reflected an over-focus on between-subject examinations and a failure to examine within-subject trial-to-trial brain-behavior relationships.

A meta-analysis of 20 between-subjects studies revealed a significant meta-effect between error signals and post-error slowing, yet five within-subjects studies revealed a significantly larger relationship (Cavanagh and Shackman, 2015). Importantly, these between subjects findings don't tell us if the ERN is actually linked to subsequent behavioral adjustment: it could be the case that, for example, a personality dimension like anxiety simply makes people more cautious in general.

In statistical terminology, within-subject single trial regressions can be considered a Level 1 analysis and betweensubjects brain-behavior regressions are a Level 2 analysis. These can be modeled together using mixed linear modeling (Singer and Willett, 2003), hierarchical linear modeling (see "Linking EEG and Behavior With Latent Variable Estimation (Hierarchical Bayesian Models)" section), or by using the regression weight of the Level 1 analysis as a first-order statistical summary for input into standard General Linear Models (i.e., as implemented in toolboxes such as SPM, Fieldtrip and LIMO EEG). This simple approach has been used to show that people with Generalized Anxiety Disorder indeed have different single trial brain-behavior relationships than controls, suggesting that (Level 2) individual differences influence (Level 1) mechanisms for instantiating cognitive control (Cavanagh et al., 2017).

In practice, the low signal to noise features of EEG lead to limited resolution of single trial activities. This can be bolstered by the approaches discussed within this review, including spatial and temporal filtering (see Debener et al., 2005), but users often apply non-parametric methods like Spearman's rank correlation coefficient in order to diminish the influence of outliers and non-linearity. Single trial multiple regression can be computed in MATLAB with ample protection against outliers using the robustfit function, which uses robust regression to down-weight outliers. Alternatively, LIMO EEG implements first and second level GLM's using robust statistical tools which emphasize effect size and which do not require preselection of peaks or components.

\section{Similarity Analysis}

One of the issues that arises in identifying relationships between unique datasets is the difference in the nature and dimensionality of each measure. We highlight RSA as a promising method for transforming different datasets into a common representational space prior to assessing their similarity (Kriegeskorte et al., 2008). Of note, RSA has been used to identify relationships among diverse types of neuroimaging datasets, such as EEG and magnetoencephalography (MEG; Su et al., 2012, 2014), MEG and fMRI (Cichy et al., 2014, 2016), ERP's and fMRI (Salmela et al., 2018), or intracranial EEG (iEEG) and fMRI (Zhang et al., 2015). Relationships among neuroimaging measures and behavior (Wardle et al., 2016), neuroimaging measures and competing 
models (Kaneshiro et al., 2015), neuroimaging measures and stimulus features (Su et al., 2014), may also be examined.

Within RSA, similarities among responses within a dataset are first derived by computing a matrix of similarities between responses across separate conditions or stimuli. This matrix, termed the representational dissimilarity matrix (RDM) may be constructed from pairwise correlation distances, pairwise classification accuracies (Cichy et al., 2016), or from confusion matrices from multi-class classifications (Kaneshiro et al., 2015). The RDM matrix represents a complexity reduced representation of the original dataset which preserves information within the original data space (i.e., by correlating fine-grained spatial or temporal activations, as opposed to the loss of information of conventional averaging; Kriegeskorte et al., 2006). By relating different RDM matrices, RSA provides a framework for identifying relationships among neuroimaging measures and behavioral data or computational models (Kriegeskorte et al., 2008).

To visualize differences between conditions, the pairwise distances among the elements within an individual RDM may be projected to two or three dimensions using multidimensional scaling (MDS; Edelman et al., 1998), and the hierarchical structure may be visualized using hierarchical clustering (Dubes and Jain, 1980). These visualization tools hold considerable promise for preserving the high-dimensional structure of these datasets, which we demonstrate using t-Distributed Stochastic Neighbor Embedding (t-SNE; van der Maaten and Hinton, 2008; Figure 6). t-SNE emphasizes the local structure of data points as opposed to the global structure, emphasized by MDS. Thus, there are different advantages to each dimension reduction approach depending on the structure of the dataset that the researcher seeks to highlight (and the nature of the data; Rieck and Leitte, 2015; Kruiger et al., 2017).

While t-SNE has been a useful visualization tool for fMRI and MRI datasets (Mwangi et al., 2014; Du et al., 2015; Mahfouz et al., 2015; Panta et al., 2016), it has been underutilized within EEG. Within Figure 6A, we demonstrate the t-SNE projection (perplexity $=25 ;$ PCA dimensions $=50$; tolerance $=0.0001)$ of single trial 64 channel ERP oddball data collected on a single healthy subject. The trials containing frequent stimuli (in black) reasonably separate from the trials containing infrequent stimuli (in red). This separation was quantified by computing the average Euclidian distance among infrequent and frequent stimuli minus the average distance among infrequent stimuli. Using this measure, we observed a greater separation between the two trial types among healthy controls ( $\mathrm{HC} ; N=58$ ) than among individuals diagnosed with schizophrenia (SZ; $N=58$; Figure 6B; bootstrap test; $p<0.001$; for participant and experiment information, see Bridwell et al., 2014). These results demonstrate that the information within single-trial EEG's may be meaningfully projected to a two-dimensional representation with t-SNE (Bridwell et al., 2018). These findings provide motivation for using visualization tools which consider the high dimensional structure of the data. However, careful consideration should be given to approaches better suited to continuous data such as EEG, and different ways to quantify
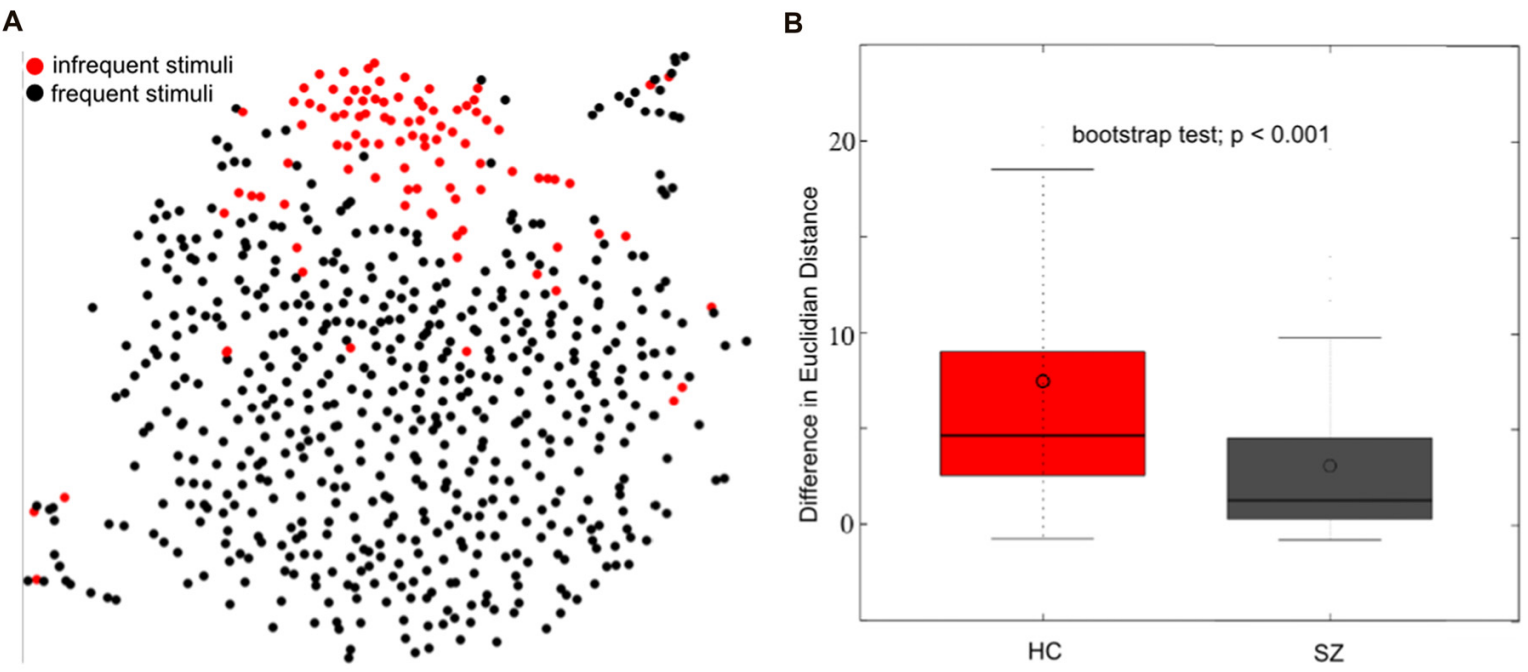

FIGURE 6 | Single trial event-related potential (ERP) projection using t-Distributed Stochastic Neighbor Embedding (t-SNE). t-SNE projects multi-dimensional datasets into a lower dimensional space for visualization. A high dimensional 654 (98 infrequent stimuli +556 frequent stimuli) $\times 12224$ (64 electrodes $\times 191$ time-point) matrix of single trial ERP's was projected to two dimensions using t-SNE (single healthy subject) in (A). The single-trial ERP responses to infrequent auditory oddball stimuli (in red) reasonably separate from the single-trial ERP responses to frequent auditory stimuli (in black), motivating the use of t-SNE for visualizing multi-electrode single-trial ERP's. The separation between frequent and infrequent stimuli was quantified as the average Euclidian distance between frequent and infrequent stimuli minus the average distance among infrequent and infrequent stimuli. A boxplot of the distribution of differences is indicated in (B) for healthy controls $(\mathrm{HC})$ and patients with schizophrenia (SZ). These results indicate a better separation among frequent and infrequent stimuli among HC than SZ (bootstrap test; $p<0.001$; modified from Bridwell et al., 2018). 
the separation of points in the lower dimensional space (e.g., Mahalanobis distance; Mahalanobis, 1936).

\section{BSS Decompositions Incorporating Multiple Datasets}

BSS extracts meaningful information from large datasets in a data-driven manner (for a review in the context of neuroimaging, see Silva et al., 2016). Focusing on a lower dimensional EEG dataset reduces the number of statistical relationships between EEG measures and external variables, which alleviates the possibility of being overly conservative in correcting for multiple statistical comparisons. This is advantageous when identifying relationships between EEG responses and behavior, as well as when examining associations between EEG and other high dimensional datasets, such as fMRI (Calhoun et al., 2009; Eichele et al., 2009; Bridwell and Calhoun, 2014).

Beyond integrating information from multiple modalities in parallel, many approaches have been developed and implemented to extract concurrent fluctuations from multiple measures in a joint decomposition, including simultaneous decompositions of EEG's and fMRI (Martínez-Montes et al., 2004; Calhoun et al., 2006, 2010; Moosmann et al., 2008; Edwards et al., 2012; Mijović et al., 2012, 2014), EEG spectra and structural MRI (sMRI; Soh et al., 2015), or EEG spectra, fMRI and sMRI (Sui et al., 2014), often with applications to clinical populations (Calhoun et al., 2009; Adali et al., 2015; Calhoun and Sui, 2016). Including all data within a multivariate multimodal decomposition isolates complex relationships between measures that may be more difficult to observe with more conventional tests, improving the ability to observe relationships between multimodal networks and behavior.

Instead of examining post hoc associations between neuroimaging measures and behavior, a more integrated approach is to decompose neuroimaging and multivariate behavioral profiles at the same time (Liu et al., 2008; Groves et al., 2011; Calhoun and Sui, 2016). Since these are two fundamentally different types of data (e.g., with different dimensions), a more flexible version of jICA, termed parallelICA, has been particularly promising. Parallel-ICA imposes constraints on the two datasets during decomposition such that correlated components are decomposed together (for details see Liu et al., 2008; Pearlson et al., 2015). For example, Meier et al., 2012 isolated a sub-component of the "resting state" network (RSN) that co-varied with behavior in a sustained attention task, and a sub-component of the left inferior frontal gyrus that co-varied with behavior during a memory task (Meier et al., 2012).

Decomposition algorithms have also been developed to decompose neuroimaging data into components which maximally differ based on a reference measure, such as single nucleotide polymorphism (SNP) allele frequencies, cognitive state estimates, or behavioral measures. The Source Power Comodulation (SPoC) approach, for example, is effective at identifying components which correlate with a target variable (e.g., correct responses, reaction time, or stimulus features), especially low SNR responses (Dähne et al., 2014).
As opposed to the standard data-driven decomposition of components, decomposition with reference imposes constraints on the decomposition such that sources are extracted which meaningfully differ based upon an external variable of interest (e.g., Liu et al., 2012).

Multi-dataset decompositions with reference have also been implemented recently by Qi et al. (2018). The authors applied "multi-site canonical correlations analysis with reference + jICA" to decompose fMRI, sMRI and dMRI data using working memory performance as a reference (Qi et al., 2018). The approach (implemented in the FIT toolbox ${ }^{2}$ ) holds considerable promise by providing a joint framework for identifying information from multiple modalities based on cognitive and behavioral differences among populations. And future studies may incorporate EEG data within these decompositions to identify differences associated with behavioral measures such as reaction times or percent correct, or latent behavioral measures such as DDM parameter estimates.

\section{Partial Least Squares}

Predictive brain-behavior relationships can be established using partial least squares (PLS) regression analyses (N-way toolbox, Andersson and Bro, 2000) of EEG. PLS is well established in neuroimaging studies (McIntosh et al., 1996; McIntosh and Lobaugh, 2004) as a method to address the problem of multicollinearity in regression models where a very large number of independent variables derived from brain data are used to model a few behavioral variables. PLS regression models estimate orthogonal components (similar to PCA) which maximize the covariance between behavioral data and the EEG data. More specifically, the PLS components are based on the optimization of a least-squares fit of a partial correlation matrix between the EEG data and dependent variables of interest (Andersson and Bro, 2000). Although PLS components lead to optimal regression models using only a few components, they are susceptible to overfitting, since the component structure is determined by the covariance with behavioral data. Thus, PLS models (as well as CCA models) must be validated by testing the prediction of new data not used in estimating the model (Huang et al., 2011; Kang et al., 2016).

Although PLS has been more extensively used in neuroimaging, a number of studies have made use of PLS models to relate EEG and evoked potential properties to individual differences in behavior. In studies of attention, PLS models have been applied to steady-state visually evoked potentials (SSVEP) responses to flickering signals and noise to account for how individual differences in the deployment of spatial attention can predict accuracy data (Krishnan et al., 2013). Similar models of the SSVEP have also been used to assess the optimal attentional filters in detection of biological motion (Hasan et al., 2017). In studies of motor learning, PLS models have been used with resting state EEG coherence to demonstrate that the functional connectivity of motor cortex can predict short-term motor learning (Wu et al., 2014). In studies of stroke patients, PLS models have shown that EEG coherence

\footnotetext{
${ }^{2}$ http://mialab.mrn.org/software/fit
} 
with motor cortex in the resting state can predict both motor impairment and improvement over a period of rehabilitation (Wu et al., 2015, 2016).

\section{Canonical Correlation Analysis}

CCA is conceptually similar to PLS (Rosipal and Krämer, 2006), except that in PLS linear structure is assumed as hidden (i.e., "latent") variables while CCA is a more nonparametric approach to finding weights that produce maximum correlation (for additional comparisons between CCA and PLS, see Sun et al., 2009; Dahne et al., 2015).

CCA transforms two multi-dimensional datasets such that the resulting variates are maximally correlated across datasets and uncorrelated within each dataset (Hotelling, 1936). The application of CCA to joint datasets consisting of EEG and behavioral or cognitive measures is limited, so we highlight select studies here which have used multi-subject extensions of CCA to identify covariations between EEG data and other neuroimaging measures such as fMRI, and sMRI (for an example linking brain connectivity patterns and demographic and psychometric measures, see Smith et al., 2015).

Multi-subject extensions of CCA (termed multimodal CCA, jCCA or mCCA) have been developed using ERP time courses and fMRI spatial maps as features (Li et al., 2009; Correa et al., 2010a). Unlike jICA, which is also implemented on multisubject multi-modal datasets, mCCA allows a separate mixing matrix for each modality and does not emphasize independence among the multimodal sources. Thus, mCCA is suitable for operating on diverse data-types with differing dimensionalities. The relationships between multi-modal feature loadings and behavioral variables such as percent-correct, confidence ratings, and average reaction time may then be examined (Correa et al., 2008, 2009, 2010a,b). Further extensions of mCCA to single trials will be useful for identifying associations in concurrently recorded ERP and fMRI datasets, and the loadings of these multi-modal variates may be related to corresponding single trial behavioral measures.

\section{LINKING EEG AND BEHAVIOR WITH LATENT VARIABLE ESTIMATION (HIERARCHICAL BAYESIAN MODELS)}

Within this section, we focus on combining EEG and behavior for simultaneously estimating latent cognitive measures. Hierarchical Bayesian modeling (HBM) of human cognition is one of the most powerful methods to integrate EEG and behavior, since these datasets are linked with respect to the cognitive function specified by the model and shared relationships are estimated simultaneously. The hierarchical Bayesian modeling (HBM) framework is ideally suited for the joint analysis of multiple modes of data (Lee, 2011; Turner et al., 2013). In addition, the EEG data can also provide new and additional information about the cognitive process that cannot be discerned with just behavior alone. This flexible framework can inform building and testing theoretical models of the relationship of electrical observations from the human cortex (EEG), human cognition and human behavior.

Bayesian inference refers to underlying probability theory and methods used to obtain conclusions about data (for an entertaining introduction to Bayesian inference see Etz and Vandekerckhove, 2017). Hierarchical modeling refers to the mathematical procedure of assuming statistical relationships between multiple levels of data description. Hierarchical modeling often yields better estimates of parameters due to shrinkage, a phenomenon whereby parameters are better estimated (and data are better described) because hierarchical relationships enforce similarity across similar parameters. For instance, the condition-level mean accuracies in an experimental task could statistically describe observed subject-level mean task accuracies through a normal distribution, and yield more predictive estimates of future subject-level ability (see Lee and Wagenmakers, 2014 for an introduction to HBMs). HBM also allows discovering complex relationships between multiple data types within cognitive neuroscience (Wiecki et al., 2015; Turner et al., 2016) by allowing simultaneous estimation of posterior distributions of multiple parameters. Fitting procedures produce samples from probability distributions that display knowledge (i.e., "uncertainty") about parameter estimates and thus certainty about the effects of cognition or EEG data in specific theoretical models.

Using the DDM of quick decision-making as an example, single-trial estimates of evidence accumulation rate during quick decision making and non-decision time (time in milliseconds of a human reaction time not related to a decision) have been obtained using hierarchical Bayesian modeling with ERP amplitude estimates on single trials, time-locked to the onset of visual stimuli. Such work is described in Nunez et al. (2017). It was found that ERP measures described trialto-trial differences in visual encoding time (a component of non-decision time during reaction time) and trial-to-trial differences in evidence accumulation rate, as described by triallevel estimates of the drift parameter (Figure 7). EEG correlates of additional cognitive processes, such as visual attention, can also add inference about the overall human cognitive process when used in combination with behavioral modeling. Nunez et al. (2015) found evidence that differences in experimental participants' attention (both visual noise suppression and visual signal enhancement) as measured by SSVEPs related to some specific differences in participants' cognition during decisionmaking.

Modern software allows HBM to be easily created, built and tested with both behavioral and EEG data using multiple types of Markov Chain Monte Carlo (MCMC) sampling techniques (see Table 2). Although still being developed and improved, JAGS (Plummer, 2003), Stan (Carpenter et al., 2017), and PyMC3 (Salvatier et al., 2016) are all recommended tools for these steps. These programs allows users to build almost any cognitive hierarchical models they choose. For example, these programs can readily be adapted to HBM of EEG and behavior using RL models. However, cognitive neuroscientific theory must be further developed to learn useful combined generative models of observed cortical dynamics, 


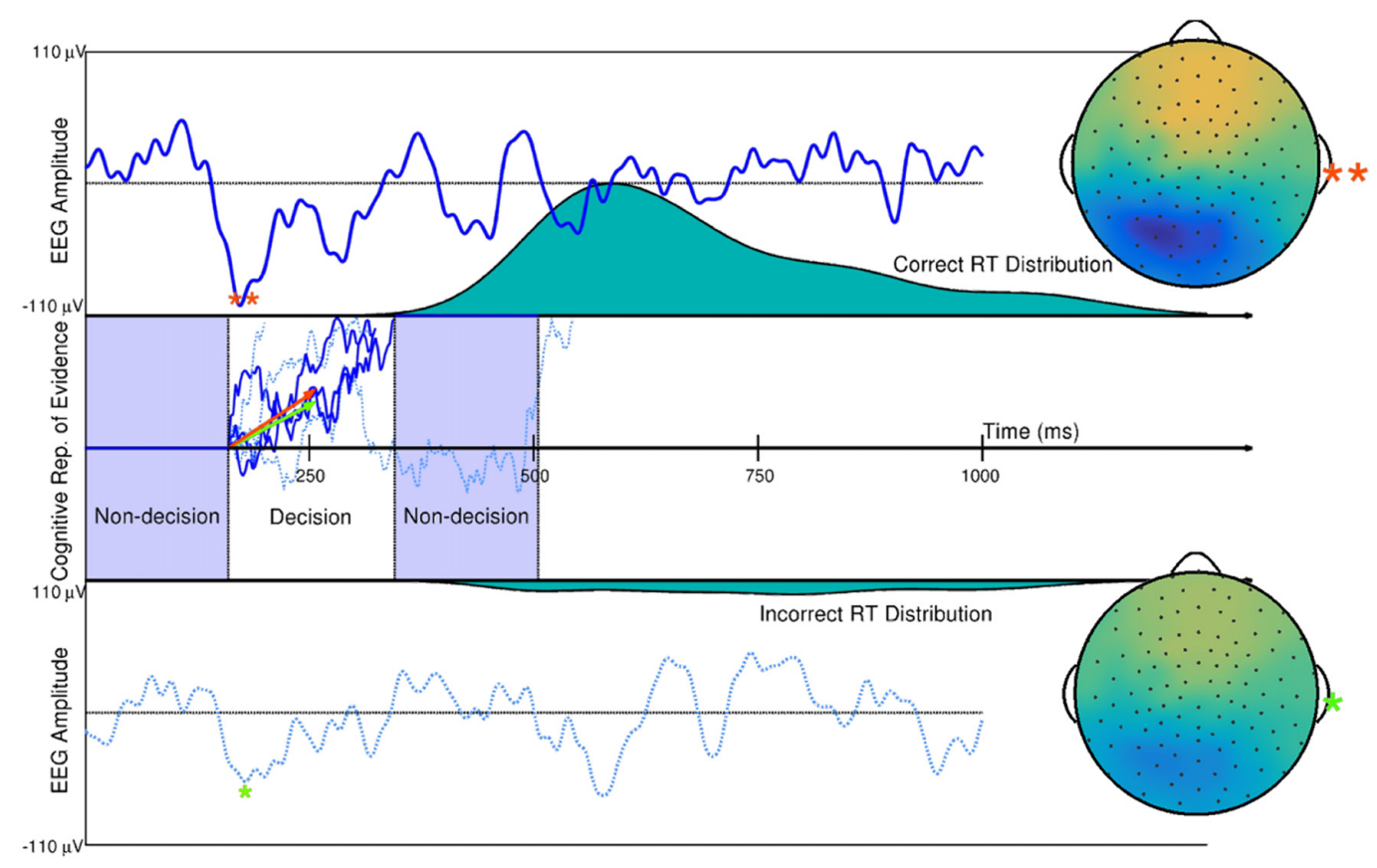

FIGURE 7 | Single trial ERP influence on DDM parameters. Two trials of a subject's spatially weight-averaged ERP are shown (top and bottom panels) along with simulations of this subject's cognitive representation of evidence (middle panel) that are derived from RT distributions. The 10th and 90th percentiles of this subject's single-trial drift rates (within-trial average evidence accumulation rates in a Brownian motion process as assumed by the DDM) are shown as the orange and green vectors. Results from hierarchical Bayesian modeling suggested that single-trial N200 amplitudes (peaks and spline-interpolated scalp maps denoted by the orange and green asterisks) influence single-trial drift rates (i.e., one latent cognitive parameter that describes the time course and latency of a decision). Using fitted parameters from real data, the larger drift rate is a linear function of the larger single-trial N200 amplitude $(* *)$, while the smaller drift rate is a linear function of the smaller N200 amplitude $\left({ }^{*}\right)$. The three dark blue evidence time courses were generated with the larger drift rate (orange vector) which is more likely to produce faster reaction times (where one path describes the time course of the example decision time and subsequent remaining non-decision time in the Middle panel). The three dotted, light blue evidence time courses were generated with the smaller drift rate (green vector) which is more likely to produce slower reaction times. True Brownian motion processes were estimated using a simple numerical technique discussed in Brown et al. (2006). Further explanation of the simulation and model fitting exists in Nunez et al. (2017).

cognition, and human behavior. Specific examples of these programs in use include (1) HDDM: python functions that can perform linear regression between calculated EEG signals on single-trials and parameters of DDMs of accuracy and reaction time data (Wiecki and Frank, 2013); (2) Hawkins et al. (2017) created $\mathrm{R}$ code and examples for sampling from hierarchical drift-diffusion models (HDDM) with neural inputs $^{3}$; and (3) MATLAB and JAGS example code performing some HDDM analyses with single-trial EEG inputs can be found at https://github.com/mdnunez/montoolbox (Nunez et al., 2017).

\section{CONCLUSION}

Neuroimaging research is focused on understanding the links between neural activity and observable behavior. This knowledge

${ }^{3}$ https://osf.io/ws3fn will help us understand how individual differences in the brain contribute to individual differences in cognitive processes. EEG is low cost, portable and provides millisecond resolution of brain activity. Thus, it is a promising tool for examining neural measures at the time scales of cognitive function. In order to relate EEG features with behavior, it will be important to obtain reliable single-trial estimates of EEG features using advanced processing and machine learning tools described within this review, and to move beyond the conventional focus on evoked components derived after averaging across trials and subjects. In addition, the accumulation of EEG depositories through data sharing and quantitative extensions beyond binary comparisons will also help advance the field. The resulting single trial EEG measures may be correlated with direct or latent measures of behavior post hoc, decomposed jointly with behavior, or integrated with behavior to derive estimates of latent variables which represent cognitive function. In doing so, we hope to

TABLE 2 | Publicly available toolboxes for (hierarchical) Bayesian parameter fitting.

\begin{tabular}{llll}
\hline Toolbox & Programming language & Citation & Link \\
\hline JAGS & Base software and Interfaces for Python, R, and MATLAB & Plummer (2003) & http://mcmc-jags.sourceforge.net/ \\
Stan & Interfaces for Python, R, MATLAB, and Julia & Carpenter et al. (2017) & http://mc-stan.org/ \\
PyMC3 & Python & Salvatier et al. (2016) & https://github.com/pymc-devs/pymc3
\end{tabular}


further our understanding of brain dynamics that contribute to moment-to-moment cognitive function.

\section{AUTHOR CONTRIBUTIONS}

DAB, JFC, AGEC, MDN, RS, SS and VDC wrote the manuscript and approved the final version.

\section{REFERENCES}

Adali, T., Levin-Schwartz, Y., and Calhoun, V. D. (2015). Multimodal data fusion using source separation: application to medical imaging. Proc. IEEE 103, 1494-1506. doi: 10.1109/jproc.2015.2461601

Andersson, C. A., and Bro, R. (2000). The $N$-way toolbox for MATLAB. Chemom. Intell. Lab. Syst. 52, 1-4. doi: 10.1016/S0169-7439(00)00071-X

Anemüller, J., Sejnowski, T. J., and Makeig, S. (2003). Complex independent component analysis of frequency-domain electroencephalographic data. Neural Netw. 16, 1311-1323. doi: 10.1016/j.neunet.2003.08.003

Basar, E. (1999). Brain Function and Oscillations, Principles and Approaches, 1. Berlin: Springer.

Bashivan, P., Rish, I., Yeasin, M., and Codella, N. (2016). "Learning representations from EEG with deep recurrent-convolutional neural networks," in Proceedings of the International Conference on Learning Representations (San Juan), 2-4.

Bendat, J. S., and Piersol, A. G. (2010). Random Data. Analysis and Measurement Procedures. 4th Edn. New York, NY: John Wiley \& Sons.

Bernat, E. M., Williams, W. J., and Gehring, W. J. (2005). Decomposing ERP time-frequency energy using PCA. Clin. Neurophysiol. 116, 1314-1334. doi: 10.1016/j.clinph.2005.01.019

Bourlard, H., and Kamp, Y. (1988). Auto-association by multilayer perceptrons and singular value decomposition. Biol. Cybern. 59, 291-294. doi: $10.1007 /$ bf00332918

Bridwell, D. A., and Calhoun, V. D. (2014). "Fusing Concurrent EEG and fMRI intrinsic networks," in Magnetoencephalography, eds S. Supek and C. Aine (Berlin: Springer-Verlag), 213-235.

Bridwell, D. A., Kiehl, K. A., Pearlson, G. D., and Calhoun, V. D. (2014). Patients with schizophrenia demonstrate reduced cortical sensitivity to auditory oddball regularities. Schizophr. Res. 158, 189-194. doi: 10.1016/j.schres.2014. 06.037

Bridwell, D. A., Liddle, B. J., Kiehl, K. A., Pearlson, G. D., and Calhoun, V. D. (2018). “T-SNE visualization of single trial oddball ERP's in controls and patients with schizophrenia," in Organization for Human Brain Mapping.

Bridwell, D. A., Leslie, E., McCoy, D., Plis, S., and Calhoun, V. D. (2017). Cortical sensitivity to guitar note patterns: EEG entrainment to repetition and key. Front. Hum. Neurosci. 11:90. doi: 10.3389/fnhum.2017.00090

Bridwell, D. A., Rachakonda, S., Silva, R. F., Pearlson, G. D., and Calhoun, V. D. (2016). Spatiospectral decomposition of multi-subject EEG: evaluating blind source separation algorithms on real and realistic simulated data. Brain Topogr. 31, 47-61. doi: 10.1007/s10548-016-0479-1

Bridwell, D. A., Steele, V. R., Maurer, J. M., Kiehl, K. A., and Calhoun, V. D. (2015). The relationship between somatic and cognitive-affective depression symptoms and error-related ERPs. J. Affect. Disord. 172, 89-95. doi: 10.1016/j.jad.2014. 09.054

Bridwell, D. A., Wu, L., Eichele, T., and Calhoun, V. D. (2013). The spatiospectral characterization of brain networks: fusing concurrent EEG spectra and fMRI maps. Neuroimage 69, 101-111. doi: 10.1016/j.neuroimage.2012.12.024

Brown, S. D., Ratcliff, R., and Smith, P. L. (2006). Evaluating methods for approximating stochastic differential equations. J. Math. Psychol. 50, 402-410. doi: $10.1016 /$ j.jmp.2006.03.004

Bugli, C., and Lambert, P. (2007). Comparison between principal component analysis and independent component analysis in electroencephalograms modelling. Biom. J. 49, 312-327. doi: 10.1002/bimj.200510285

Calhoun, V., and Adali, T. (2012). Multi-subject independent component analysis of fMRI: a decade of intrinsic networks, default mode and neurodiagnostic discovery. IEEE Rev. Biomed. Eng. 5, 60-73. doi: 10.1109/rbme.2012.22 11076

\section{FUNDING}

This work was supported by grants from the National Institutes of Health 2R01EB005846, R01REB020407 and P20GM103472 to VDC; and National Science Foundation grant number 1539067 and 1658303.

Calhoun, V. D., Adali, T., Pearlson, G. D., and Kiehl, K. A. (2006). Neuronal chronometry of target detection: fusion of hemodynamic and event-related potential data. Neuroimage 30, 544-553. doi: 10.1016/j.neuroimage.2005. 08.060

Calhoun, V. D., Adali, T., Pearlson, G. D., and Pekar, J. J. (2001). A method for making group inferences from functional MRI data using independent component analysis. Hum. Brain Mapp. 14, 140-151. doi: 10.1002/hbm.1048

Calhoun, V. D., Liu, J., and Adali, T. (2009). A review of group ICA for fMRI data and ICA for joint inference of imaging, genetic and ERP data. Neuroimage 45, S163-S172. doi: 10.1016/j.neuroimage.2008.10.057

Calhoun, V. D., and Sui, J. (2016). Multimodal fusion of brain imaging data: a key to finding the missing link(s) in complex mental illness. Biol. Psychiatry Cogn. Neurosci. Neuroimaging 1, 230-244. doi: 10.1016/j.bpsc.2015.12.005

Calhoun, V. D., Wu, L., Kiehl, K. A., Eichele, T., and Pearlson, G. D. (2010). Aberrent processing of deviant stimuli in schizophrenia revealed by fusion of fMRI and EEG data. Acta Neuropsychiatr. 22, 127-138. doi: 10.1111/j.16015215.2010.00467.x

Carpenter, B., Gelman, A., Hoffman, M. D., Lee, D., Goodrich, B., Betancourt, M., et al. (2017). Stan: a probabilistic programming language. J. Stat. Softw. 76:1. doi: $10.18637 /$ jss.v076.i01

Cavanagh, J. F., Meyer, A., and Hajcak, G. (2017). Error-specific cognitive control alterations in generalized anxiety disorder. Biol. Psychiatry Cogn. Neurosci. Neuroimaging 2, 413-420. doi: 10.1016/j.bpsc.2017.01.004

Cavanagh, J. F., and Shackman, A. J. (2015). Frontal midline theta reflects anxiety and cognitive control: meta-analytic evidence. J. Physiol. Paris 109, 3-15. doi: 10.1016/j.jphysparis.2014.04.003

Cavanagh, J. F., Wiecki, T. V., Cohen, M. X., Figueroa, C. M., Samanta, J., Sherman, S. J., et al. (2011). Subthalamic nucleus stimulation reverses mediofrontal influence over decision threshold. Nat. Neurosci. 14, 1462-1467. doi: 10.1038/nn.2925

Cichy, R. M., Pantazis, D., and Oliva, A. (2014). Resolving human object recognition in space and time. Nat. Neurosci. 17, 455-462. doi: 10.1038/nn.3635

Cichy, R. M., Pantazis, D., and Oliva, A. (2016). Similarity-based fusion of MEG and fmri reveals spatio-temporal dynamics in human cortex during visual object recognition. Cereb. Cortex 26, 3563-3579. doi: 10.1093/cercor/bhw135

Cohen, M. X. (2014). Analyzing Neural Time Series Data. Cambridge, MA: The MIT Press.

Cohen, M. X., and Cavanagh, J. F. (2011). Single-trial regression elucidates the role of prefrontal theta oscillations in response conflict. Front. Psychol. 2:30. doi: 10.3389/fpsyg.2011.00030

Cole, S. R., van der Meij, R., Peterson, E. J., de Hemptinne, C., Starr, P. A., and Voytek, B. (2017). Nonsinusoidal $\beta$ oscillations reflect cortical pathophysiology in Parkinson's disease. J. Neurosci. 37, 4830-4840. doi: 10.1523/jneurosci.220816.2017

Cole, S. R., and Voytek, B. (2017). Brain oscillations and the importance of waveform shape. Trends Cogn. Sci. 21, 137-149. doi: 10.1016/j.tics.2016.12.008

Collins, A. G. E., Cavanagh, J. F., and Frank, M. J. (2014). Human EEG uncovers latent generalizable rule structure during learning. J. Neurosci. 34, 4677-4685. doi: 10.1523/JNEUROSCI.3900-13.2014

Collins, A. G., and Frank, M. J. (2016). Neural signature of hierarchically structured expectations predicts clustering and transfer of rule sets in reinforcement learning. Cognition 152, 160-169. doi: 10.1016/j.cognition.2016. 04.002

Cong, F., He, Z., Hämäläinen, J., Leppänen, P. H. T., Lyytinen, H., Cichocki, A., et al. (2013). Validating rationale of group-level component analysis based on estimating number of sources in EEG through model order selection. J. Neurosci. Methods 212, 165-172. doi: 10.1016/j.jneumeth.2012. 09.029 
Cong, F., Lin, Q.-H., Kuang, L.-D., Gong, X.-F., Astikainen, P., and Ristaniemi, T. (2015). Tensor decomposition of EEG signals: a brief review. J. Neurosci. Methods 248, 59-69. doi: 10.1016/j.jneumeth.2015.03.018

Congedo, M., John, R. E., De Ridder, D., and Prichep, L. (2010). Group independent component analysis of resting state EEG in large normative samples. Int. J. Psychophysiol. 78, 89-99. doi: 10.1016/j.ijpsycho.2010.06.003

Correa, N. M., Eichele, T., Adali, T., Li, Y.-O., and Calhoun, V. D. (2010a). "Fusion of concurrent single trial EEG data and fMRI data using multi-set canonical correlation analysis," in Proceedings of the IEEE International Conference on Acoustics Speech and Signal Processing (ICASSP) (Dallas: IEEE) 5438-5441. Available online at: http://ieeexplore.ieee.org/xpls/abs_all. jsp? arnumber $=5494919$

Correa, N. M., Eichele, T., Adali, T., Li, Y.-O., and Calhoun, V. D. (2010b). Multiset canonical correlation analysis for the fusion of concurrent single trial ERP and functional MRI. Neuroimage 50, 1438-1445. doi: 10.1016/j.neuroimage. 2010.01.062

Correa, N. M., Li, Y.-O., Adali, T., and Calhoun, V. D. (2008). Canonical correlation analysis for feature-based fusion of biomedical imaging modalities and its application to detection of associative networks in schizophrenia. IEEE J. Sel. Top. Signal Process. 2, 998-1007. doi: 10.1109/jstsp.2008. 2008265

Correa, N. M., Li, Y.-O., Adali, T., and Calhoun, V. D. (2009). "Fusion of fMRI, sMRI, and EEG data using canonical correlation analysis," in Proceedings of the IEEE International Conference on Acoustics, Speech and Signal Processing (ICASSP) (Taipei: IEEE), 385-388. Available online at: http://ieeexplore.ieee.org/abstract/document/4959601/

Dahne, S., Bieszmann, F., Samek, W., Haufe, S., Goltz, D., Gundlach, C., et al. (2015). Multivariate machine learning methods for fusing multimodal functional neuroimaging data. Proc. IEEE 103, 1507-1530. doi: 10.1109/jproc. 2015.2425807

Dähne, S., Meinecke, F. C., Haufe, S., Höhne, J., Tangermann, M., Müller, K.-R., et al. (2014). SPoC: a novel framework for relating the amplitude of neuronal oscillations to behaviorally relevant parameters. Neuroimage 86, 111-122. doi: 10.1016/j.neuroimage.2013.07.079

Debener, S., Ullsperger, M., Siegel, M., Fiehler, K., von Cramon, D. Y., and Engel, A. K. (2005). Trial-by-trial coupling of concurrent electroencephalogram and functional magnetic resonance imaging identifies the dynamics of performance monitoring. J. Neurosci. 25, 11730-11737. doi: 10.1523/JNEUROSCI.3286-05.2005

Delorme, A., and Makeig, S. (2004). EEGLAB: an open source toolbox for analysis of single-trial EEG dynamics including independent component analysis. J. Neurosci. Methods 134, 9-21. doi: 10.1016/j.jneumeth.2003.10.009

Dien, J. (2010). The ERP PCA Toolkit: an open source program for advanced statistical analysis of event-related potential data. J. Neurosci. Methods 187, 138-145. doi: 10.1016/j.jneumeth.2009.12.009

Dien, J., Beal, D. J., and Berg, P. (2005). Optimizing principal components analysis of event-related potentials: matrix type, factor loading weighting, extraction, and rotations. Clin. Neurophysiol. 116, 1808-1825. doi: 10.1016/j.clinph.2004. 11.025

Dien, J., Khoe, W., and Mangun, G. R. (2007). Evaluation of PCA and ICA of simulated ERPs: promax vs. Hum. Brain Mapp. 28, 742-763. doi: 10.1002/hbm. 20304

Donchin, E., and Coles, M. G. (1988). Is the P300 component a manifestation of context updating? Behav. Brain Sci. 11, 357-374. doi: 10.1017/s0140525x0005 8027

Donchin, E., Ritter, W., and McCallum, W. C. (1978). "Cognitive psychophysiology: The endogenous components of the ERP," in Event Related Brain Potentials in Man, eds E. Callaway, P. Tueting and S. H. Joslow (New York, NY: Academic Press), 349-441.

Du, Y., Pearlson, G. D., Liu, J., Sui, J., Yu, Q., He, H., et al. (2015). A group ICA based framework for evaluating resting fMRI markers when disease categories are unclear: application to schizophrenia, bipolar and schizoaffective disorders. Neuroimage 122, 272-280. doi: 10.1016/j.neuroimage.2015.07.054

Dubes, R., and Jain, A. K. (1980). Clustering methodologies in exploratory data analysis. Adv. Comput. 19, 113-228. doi: 10.1016/s0065-2458(08)60034-0

Edelman, S., Grill-Spector, K., Kushnir, T., and Malach, R. (1998). Toward direct visualization of the internal shape representation space by fMRI. Psychobiology 26, 309-321.
Edwards, B. G., Calhoun, V. D., and Kiehl, K. A. (2012). Joint ICA of ERP and fMRI during error-monitoring. Neuroimage 59, 1896-1903. doi: 10.1016/j. neuroimage.2011.08.088

Eichele, T., Calhoun, V. D., and Debener, S. (2009). Mining EEG-fMRI using independent component analysis. Int. J. Psychophysiol. 73, 53-61. doi: 10.1016/j.ijpsycho.2008.12.018

Eichele, T., Rachakonda, S., Brakedal, B., Eikeland, R., and Calhoun, V. D. (2011). EEGIFT: group independent component analysis for event-related EEG data. Comput. Intell. Neurosci. 2011:129365. doi: 10.1155/2011/ 129365

Etz, A., and Vandekerckhove, J. (2017). Introduction to bayesian inference for psychology. Psychon. Bull. Rev. doi: 10.3758/s13423-017-1262-3 [Epub ahead of print].

Falkenstein, M., Hohnsbein, J., Hoormann, J., and Blanke, L. (1991). Effects of crossmodal divided attention on late ERP components. II. Error processing in choice reaction tasks. Electroencephalogr. Clin. Neurophysiol. 78, 447-455. doi: 10.1016/0013-4694(91)90062-9

Folstein, J. R., and Van Petten, C. (2007). Influence of cognitive control and mismatch on the N2 component of the ERP: a review. Psychophysiology 45, 152-170. doi: 10.1111/j.1469-8986.2007.00602.x

Frank, M. J., Gagne, C., Nyhus, E., Masters, S., Wiecki, T. V., Cavanagh, J. F., et al. (2015). fMRI and EEG predictors of dynamic decision parameters during human reinforcement learning. J. Neurosci. 35, 485-494. doi: 10.1523/JNEUROSCI.2036-14.2015

Frank, M. J., Seeberger, L. C., and O'Reilly, R. (2004). By carrot or by stick: cognitive reinforcement learning in parkinsonism. Science 306, 1940-1943. doi: $10.1126 /$ science. 1102941

Fries, P. (2005). A mechanism for cognitive dynamics: neuronal communication through neuronal coherence. Trends Cogn. Sci. 9, 474-480. doi: 10.1016/j.tics. 2005.08.011

Gaspar, C. M., Rousselet, G. A., and Pernet, C. R. (2011). Reliability of ERP and single-trial analyses. Neuroimage 58, 620-629. doi: 10.1016/j.neuroimage.2011. 06.052

Gehring, W. J., Goss, B., Coles, M. G. H., Meyer, D. E., and Donchin, E. (1993). A neural system for error detection and compensation. Psychol. Sci. 4, 385-390. doi: 10.1111/j.1467-9280.1993.tb00586.x

Groves, A. R., Beckmann, C. F., Smith, S. M., and Woolrich, M. W. (2011). Linked independent component analysis for multimodal data fusion. Neuroimage 54, 2198-2217. doi: 10.1016/j.neuroimage.2010.09.073

Harmony, T. (2013). The functional significance of delta oscillations in cognitive processing. Front. Integr. Neurosci. 7:83. doi: 10.3389/fnint.2013.00083

Hasan, R., Srinivasan, R., and Grossman, E. D. (2017). Feature-based attentional tuning during biological motion detection measured with SSVEP. J. Vis. 17:22. doi: $10.1167 / 17.9 .22$

Hawkins, G. E., Mittner, M., Forstmann, B. U., and Heathcote, A. (2017). On the efficiency of neurally-informed cognitive models to identify latent cognitive states. J. Math. Psychol. 76, 142-155. doi: 10.1016/j.jmp.2016. 06.007

Hotelling, H. (1936). Relations between two sets of variates. Biometrika 28, 321-377. doi: 10.2307/2333955

Huang, X., Qin, G., and Fang, Y. (2011). Optimal combinations of diagnostic tests based on AUC. Biometrics 67, 568-576. doi: 10.1111/j.1541-0420.2010. 01450.x

Huster, R. J., Plis, S. M., and Calhoun, V. D. (2015). Group-level component analyses of EEG: validation and evaluation. Front. Neurosci. 9:254. doi: 10.3389/fnins.2015.00254

Huster, R. J., and Raud, L. (2018). A tutorial review on multi-subject decomposition of EEG. Brain Topogr. 31, 3-16. doi: 10.1007/s10548-017 $-0603-\mathrm{X}$

Huys, Q. J. M., Maia, T. V., and Frank, M. J. (2016). Computational psychiatry as a bridge from neuroscience to clinical applications. Nat. Neurosci. 19, 404-413. doi: $10.1038 / \mathrm{nn} .4238$

Hyvarinen, A., Karhunen, J., and Oja, E. (2001). Independent Component Analysis. New York, NY: John Wiley and Sons.

Hyvärinen, A., Ramkumar, P., Parkkonen, L., and Hari, R. (2010). Independent component analysis of short-time Fourier transforms for spontaneous EEG/MEG analysis. Neuroimage 49, 257-271. doi: 10.1016/j.neuroimage.2009. 08.028 
Jensen, O., Kaiser, J., and Lachaux, J.-P. (2007). Human $\gamma$-frequency oscillations associated with attention and memory. Trends Neurosci. 30, 317-324. doi: 10.1016/j.tins.2007.05.001

Jones, S. R. (2016). When brain rhythms aren't 'rhythmic': implication for their mechanisms and meaning. Curr. Opin. Neurobiol. 40, 72-80. doi: 10.1016/j. conb.2016.06.010

Jung, T. P., Makeig, S., Humphries, C., Lee, T. W., McKeown, M. J., Iragui, V., et al. (2000). Removing electroencephalographic artifacts by blind source separation. Psychophysiology 37, 163-178. doi: 10.1111/1469-8986.3720163

Kaneshiro, B., Perreau Guimaraes, M., Kim, H.-S., Norcia, A. M., and Suppes, P. (2015). A representational similarity analysis of the dynamics of object processing using single-trial EEG classification. PLoS One 10:e135697. doi: 10.1371/journal.pone.0135697

Kang, L., Liu, A., and Tian, L. (2016). Linear combination methods to improve diagnostic/prognostic accuracy on future observations. Stat. Methods Med. Res. 25, 1359-1380. doi: 10.1177/0962280213481053

Kayser, J., and Tenke, C. E. (2003). Optimizing PCA methodology for ERP component identification and measurement: theoretical rationale and empirical evaluation. Clin. Neurophysiol. 114, 2307-2325. doi: 10.1016/s13882457(03)00241-4

Klimesch, W. (1999). EEG $\alpha$ and theta oscillations reflect cognitive and memory performance: a review and analysis. Brain Res. Rev. 29, 169-195. doi: 10.1016/s0165-0173(98)00056-3

Kovacevic, N., and McIntosh, A. R. (2007). Groupwise independent component decomposition of EEG data and partial least square analysis. Neuroimage 35, 1103-1112. doi: 10.1016/j.neuroimage.2007.01.016

Kriegeskorte, N., Goebel, R., and Bandettini, P. (2006). Information-based functional brain mapping. Proc. Natl. Acad. Sci. U S A 103, 3863-3868. doi: 10.1073/pnas.0600244103

Kriegeskorte, N., Mur, M., and Bandettini, P. (2008). Representational similarity analysis-connecting the branches of systems neuroscience. Front. Syst. Neurosci. 2:4. doi: 10.3389/neuro.06.004.2008

Krishnan, L., Kang, A., Sperling, G., and Srinivasan, R. (2013). Neural strategies for selective attention distinguish fast-action video game players. Brain Topogr. 26, 83-97. doi: 10.1007/s10548-012-0232-3

Kruiger, J. F., Rauber, P. E., Martins, R. M., Kerren, A., Kobourov, S., and Telea, A. C. (2017). Graph layouts by t-SNE. Comput. Graph. Forum. 36, 283-294. doi: 10.1111/cgf.13187

Labounek, R., Bridwell, D. A., Mareček, R., Lamoš, M., Mikl, M., Slavíček, T., et al. (2018). Stable scalp EEG spatiospectral patterns across paradigms estimated by group ICA. Brain Topogr. 31, 76-89. doi: 10.1007/s10548-017-0585-8

Lachaux, J. P., Lutz, A., Rudrauf, D., Cosmelli, D., Le Van Quyen, M., Martinerie, J., et al. (2002). Estimating the time-course of coherence between single-trial brain signals: an introduction to wavelet coherence. Neurophysiol. Clin. 32, 157-174. doi: 10.1016/s0987-7053(02)00301-5

Laming, D. R. J. (1979). Autocorrelation of choice-reaction times. Acta Psychol. 43, 381-412. doi: 10.1016/0001-6918(79)90032-5

Le Van Quyen, M., Foucher, J., Lachaux, J.-P., Rodriguez, E., Lutz, A., Martinerie, J., et al. (2001). Comparison of Hilbert transform and wavelet methods for the analysis of neuronal synchrony. J. Neurosci. Methods 111, 83-98. doi: 10.1016/s0165-0270(01)00372-7

Lee, M. D. (2011). How cognitive modeling can benefit from hierarchical Bayesian models. J. Math. Psychol. 55, 1-7. doi: 10.1016/j.jmp.2010.08.013

Lee, M. D., and Wagenmakers, E. J. (2014). Bayesian Cognitive Modeling: A Practical Course. Boston, MA: Cambridge University Press.

Li, Y. O., Adali, T., Wang, W., and Calhoun, V. D. (2009). Joint blind source separation by multiset canonical correlation analysis. IEEE Trans. Signal. Process. 57, 3918-3929. doi: 10.1109/TSP.2009.2021636

Lio, G., and Boulinguez, P. (2013). Greater robustness of second order statistics than higher order statistics algorithms to distortions of the mixing matrix in blind source separation of human EEG: implications for single-subject and group analyses. Neuroimage 67, 137-152. doi: 10.1016/j.neuroimage. 2012.11.015

Liu, J., Demirci, O., and Calhoun, V. D. (2008). A parallel independent component analysis approach to investigate genomic influence on brain function. IEEE Signal Process. Lett. 15, 413-416. doi: 10.1109/lsp.2008.922513

Liu, J., Ghassemi, M. M., Michael, A. M., Boutte, D., Wells, W., PerroneBizzozero, N., et al. (2012). An ICA with reference approach in identification of genetic variation and associated brain networks. Front. Hum. Neurosci. 6:21. doi: 10.3389/fnhum.2012.00021

Luck, S. (2014). An Introduction to the Event-Related Potential Technique. 2nd Edn. Boston, MA: MIT Press.

Luck, S. J., and Kappenman, E. S. (2012). The Oxford Handbook of Event-Related Potential Components. New York, NY: Oxford University Press.

Mahalanobis, P. C. (1936). On the generalized distance in statistics. Proc. Natl. Inst. Sci. India 2, 49-55.

Mahfouz, A., van de Giessen, M., van der Maaten, L., Huisman, S., Reinders, M., Hawrylycz, M. J., et al. (2015). Visualizing the spatial gene expression organization in the brain through non-linear similarity embeddings. Methods 73, 79-89. doi: 10.1016/j.ymeth.2014.10.004

Makeig, S., Debener, S., Onton, J., and Delorme, A. (2004). Mining event-related brain dynamics. Trends Cogn. Sci. 8, 204-210. doi: 10.1016/j.tics.2004.03.008

Makeig, S., Jung, T.-P., Bell, A. J., Ghahremani, D., and Sejnowski, T. J. (1997). Blind separation of auditory event-related brain responses into independent components. Proc. Natl. Acad. Sci. U S A 94, 10979-10984. doi: 10.1073/pnas. 94.20.10979

Makeig, S., Westerfield, M., Jung, T. P., Enghoff, S., Townsend, J., Courchesne, E., et al. (2002). Dynamic brain sources of visual evoked responses. Science 295, 690-694. doi: 10.1126/science. 1066168

Martínez-Montes, E., Valdés-Sosa, P. A., Miwakeichi, F., Goldman, R. I., and Cohen, M. S. (2004). Concurrent EEG/fMRI analysis by multiway partial least squares. Neuroimage 22, 1023-1034. doi: 10.1016/j.neuroimage.2004. 03.038

McIntosh, A. R., and Lobaugh, N. J. (2004). Partial least squares analysis of neuroimaging data: applications and advances. Neuroimage 23, S250-S263. doi: 10.1016/j.neuroimage.2004.07.020

McIntosh, A. R., Bookstein, F. L., Haxby, J. V., and Grady, C. L. (1996). Spatial pattern analysis of functional brain images using partial least squares. Neuroimage 3, 143-157. doi: 10.1006/nimg.1996.0016

Meier, T. B., Wildenberg, J. C., Liu, J., Chen, J., Calhoun, V. D., Biswal, B. B., et al. (2012). Parallel ICA identifies sub-components of resting state networks that covary with behavioral indices. Front. Hum. Neurosci. 6:281. doi: 10.3389/fnhum.2012.00281

Mijović, B., De Vos, M., Vanderperren, K., Machilsen, B., Sunaert, S., Van Huffel, S., et al. (2014). The dynamics of contour integration: a simultaneous EEG-fMRI study. Neuroimage 88, 10-21. doi: 10.1016/j.neuroimage.2013. 11.032

Mijović, B., Vanderperren, K., Novitskiy, N., Vanrumste, B., Stiers, P., Van den Bergh, B., et al. (2012). The "why" and "how" of JointICA: results from a visual detection task. Neuroimage 60, 1171-1185. doi: 10.1016/j.neuroimage.2012. 01.063

Montague, P. R., Dolan, R. J., Friston, K. J., and Dayan, P. (2012). Computational psychiatry. Trends Cogn. Sci. 16, 72-80. doi: 10.1016/j.tics.2011.11.018

Moosmann, M., Eichele, T., Nordby, H., Hugdahl, K., and Calhoun, V. D. (2008). Joint independent component analysis for simultaneous EEG-fMRI: principle and simulation. Int. J. Psychophysiol. 67, 212-221. doi: 10.1016/j.ijpsycho.2007. 05.016

Mwangi, B., Soares, J. C., and Hasan, K. M. (2014). Visualization and unsupervised predictive clustering of high-dimensional multimodal neuroimaging data. J. Neurosci. Methods 236, 19-25. doi: 10.1016/j.jneumeth.2014.08.001

Nassar, M. R., and Frank, M. J. (2016). Taming the beast: extracting generalizable knowledge from computational models of cognition. Curr. Opin. Behav. Sci. 11, 49-54. doi: 10.1016/j.cobeha.2016.04.003

Niedermeyer, E. (1997). $\alpha$ rhythms as physiological and abnormal phenomena. Int. J. Psychophysiol. 26, 31-49. doi: 10.1016/s0167-8760(97)00754-x

Nikulin, V. V., Nolte, G., and Curio, G. (2011). A novel method for reliable and fast extraction of neuronal EEG/MEG oscillations on the basis of spatiospectral decomposition. Neuroimage 55, 1528-1535. doi: 10.1055/s-0031-12 72799

Nunez, P., and Srinivasan, R. (2006). Electric Fields of the Brain: The Neurophysics of EEG. 2nd Edn. New York, NY: Oxford University Press.

Nunez, M. D., Srinivasan, R., and Vandekerckhove, J. (2015). Individual differences in attention influence perceptual decision making. Front. Psychol. 8:18. doi: 10.3389/fpsyg.2015.00018

Nunez, M. D., Vandekerckhove, J., and Srinivasan, R. (2017). How attention influences perceptual decision making: single-trial EEG correlates of drift- 
diffusion model parameters. J. Math. Psychol. 76, 117-130. doi: 10.1016/j.jmp. 2016.03.003

Nunez, P., Wingeier, B. M., and Silbertein, R. B. (2001). Spatial-temporal structures of human $\alpha$ rhythms: theory, microcurrent sources, multiscale measurements, and global binding of local networks. Hum. Brain Mapp. 13, 125-164. doi: 10.1002/hbm.1030

Nyhus, E., and Curran, T. (2010). Functional role of $\gamma$ and theta oscillations in episodic memory. Neurosci. Biobehav. Rev. 34, 1023-1035. doi: 10.1016/j. neubiorev.2009.12.014

Onton, J., Westerfield, M., Townsend, J., and Makeig, S. (2006). Imaging human EEG dynamics using independent component analysis. Neurosci. Biobehav. Rev. 30, 808-822. doi: 10.1016/j.neubiorev.2006.06.007

Panta, S. R., Wang, R., Fries, J., Kalyanam, R., Speer, N., Banich, M., et al. (2016). A tool for interactive data visualization: application to over 10,000 brain imaging and phantom MRI data sets. Front. Neuroinform. 10:9. doi: 10.3389/fninf.2016. 00009

Pearlson, G. D., Liu, J., and Calhoun, V. D. (2015). An introductory review of parallel independent component analysis (p-ICA) and a guide to applying p-ICA to genetic data and imaging phenotypes to identify disease-associated biological pathways and systems in common complex disorders. Front. Genet. 6:276. doi: 10.3389 /fgene.2015.00276

Pernet, C. R., Chauveau, N., Gaspar, C., and Rousselet, G. A. (2011). LIMO EEG: a toolbox for hierarchical LInear MOdeling of ElectroEncephaloGraphic data. Comput. Intell. Neurosci. 2011:831409. doi: 10.1155/2011/831409

Plis, S. M., Hjelm, D. R., Salakhutdinov, R., Allen, E. A., Bockholt, H. J., Long, J. D., et al. (2014). Deep learning for neuroimaging: a validation study. Front. Neurosci. 8:229. doi: 10.3389/fnins.2014.00229

Plummer, M. (2003). "JAGS: a program for analysis of Bayesian graphical models using Gibbs sampling," in Proceedings of the 3rd International Workshop on Distributed Statistical Computing (Vienna), 125. Available online at: http://www.ci.tuwien.ac.at/Conferences/DSC-2003/Drafts/Plummer.pdf

Ponomarev, V. A., Mueller, A., Candrian, G., Grin-Yatsenko, V. A., and Kropotov, J. D. (2014). Group independent component analysis (gICA) and current source density (CSD) in the study of EEG in ADHD adults. Clin. Neurophysiol. 125, 83-97. doi: 10.1016/j.clinph.2013.06.015

Porcaro, C., Ostwald, D., and Bagshaw, A. P. (2010). Functional source separation improves the quality of single trial visual evoked potentials recorded during concurrent EEG-fMRI. Neuroimage 1, 112-123. doi: 10.1016/j.neuroimage. 2009.12.002

Qi, S., Calhoun, V. D., van Erp, T. G. M., Bustillo, J., Damaraju, E., Turner, J. A., et al. (2018). Multimodal fusion with reference: searching for joint neuromarkers of working memory deficits in schizophrenia. IEEE Trans. Med. Imaging 37, 93-105. doi: 10.1109/TMI.2017.2725306

Ramkumar, P., Parkkonen, L., and Hyvärinen, A. (2014). Group-level spatial independent component analysis of Fourier envelopes of resting-state MEG data. Neuroimage 86, 480-491. doi: 10.1016/j.neuroimage.2013. 10.032

Ratcliff, R. (1978). A theory of memory retrieval. Psychol. Rev. 85, 59-108. doi: 10.1037/0033-295X.85.2.59

Ratcliff, R., and McKoon, G. (2008). The diffusion decision model: theory and data for two-choice decision tasks. Neural Comput. 20, 873-922. doi: 10.1162/neco. 2008.12-06-420

Regan, D. (1989). Human Brian Electrophysiology: Evoked Potentials and Evoked Magnatic Fields in Science and Medicine. New York, NY: Elsevier.

Rieck, B., and Leitte, H. (2015). Persistent homology for the evaluation of dimensionality reduction schemes. Comput. Graph. Forum. 34, 431-440. doi: $10.1111 /$ cgf. 12655

Roitman, J. D., and Shadlen, M. N. (2002). Response of neurons in the lateral intraparietal area during a combined visual discrimination reaction time task. J. Neurosci. 22, 9475-9489.

Rosipal, R., and Krämer, N. (2006). Overview and recent advances in partial least squares. Lect. Notes Comput. Sci. 3940, 34-51. doi: 10.1007/11752790_2

Rousselet, G. A., and Pernet, C. R. (2011). Quantifying the time course of visual object processing using ERPs: it's time to up the game. Front. Psychol. 2:107. doi: 10.3389/fpsyg.2011.00107

Salmela, V., Salo, E., Salmi, J., and Alho, K. (2018). Spatiotemporal dynamics of attention networks revealed by representational similarity analysis of EEG and fMRI. Cereb. Cortex 28, 549-560. doi: 10.1093/cercor/bhw389
Salvatier, J., Wiecki, T. V., and Fonnesbeck, C. (2016). Probabilistic programming in Python using PyMC3. PeerJ Comput. Sci. 2:e55. doi: 10.7717/ peerj-cs. 55

Shou, G., Ding, L., and Dasari, D. (2012). Probing neural activations from continuous EEG in a real-world task: time-frequency independent component analysis. J. Neurosci. Methods 209, 22-34. doi: 10.1016/j.jneumeth.2012. 05.022

Silva, R. F., Plis, S. M., Sui, J., Pattichis, M. S., Adali, T., and Calhoun, V. D. (2016). Blind source separation for unimodal and multimodal brain networks: a unifying framework for subspace modeling. IEEE J. Sel. Top. Signal Process. 10, 1134-1149. doi: 10.1109/JSTSP.2016.2594945

Singer, J., and Willett, J. B. (2003). Applied Longitudinal Data Analysis. Oxford: Oxford University Press.

Smith, S. M., Nichols, T. E., Vidaurre, D., Winkler, A. M., Behrens, T. E. J., Glasser, M. F., et al. (2015). A positive-negative mode of population covariation links brain connectivity, demographics and behavior. Nat. Neurosci. 18, 1565-1567. doi: 10.1038/nn.4125

Soh, P., Narayanan, B., Khadka, S., Calhoun, V. D., Keshavan, M. S., Tamminga, C. A., et al. (2015). Joint coupling of awake EEG frequency activity and MRI gray matter volumes in the psychosis dimension: a BSNIP study. Front. Psychiatry 6:162. doi: 10.3389/fpsyt.2015.00162

Stober, S. (2017). "Learning discriminative features from electroencephalography recordings by encoding similarity constraints," in Proceedings of the IEEE International Conference on Acoustics, Speech and Signal Processing (ICASSP) (New Orleans: IEEE), 6175-6179. Available online at: http://ieeexplore.ieee.org/abstract/document/7953343/

Stone, J. V. (2004). Independent Component Analysis: a Tutorial Introduction. Cambridge, MA: MIT press.

Sturm, B. L. (2014). A simple method to determine if a music information retrieval system is a "horse". IEEE Trans. Multimedia 16, 1636-1644. doi: 10.1109/tmm. 2014.2330697

Su, L., Fonteneau, E., Marslen-Wilson, W., and Kriegeskorte, N. (2012). "Spatiotemporal searchlight representational similarity analysis in EMEG source space," in Proceedings of Pattern Recognition in NeuroImaging (PRNI) (London: IEEE), 97-100. doi: 10.1109/PRNI.2012.26

Su, L., Zulfiqar, I., Jamshed, F., Fonteneau, E., and Marslen-Wilson, W. (2014). Mapping tonotopic organization in human temporal cortex: representational similarity analysis in EMEG source space. Front. Neurosci. 8:368. doi: 10.3389/fnins.2014.00368

Sui, J., Castro, E., He, H., Bridwell, D., Du, Y., Pearlson, G. D., et al. (2014). "Combination of FMRI-SMRI-EEG data improves discrimination of schizophrenia patients by ensemble feature selection," in Proceedings of the 36th Annual International Conference of the IEEE Engineering in Medicine and Biology Society (EMBC) (Chicago: IEEE), 3889-3892. Available online at: http://ieeexplore.ieee.org/abstract/document/6944473/

Sun, L., Ji, S., Yu, S., and Ye, J. (2009). "On the equivalence between canonical correlation analysis and orthonormalized partial least squares," in Proceedings of the 21st International Jont Conference on Artifical Intelligence (IJCAI) (San Francisco), 1230-1235. Available online at: http://www.aaai.org/ocs/index.php/IJCAI/IJCAI-09/paper/download/352/968

Sutton, R. S., and Barto, A. G. (1998). Reinforcement Learning: an Introduction. Cambridge, MA: The MIT Press.

Turner, B. M., Forstmann, B. U., Wagenmakers, E.-J., Brown, S. D., Sederberg, P. B., and Steyvers, M. (2013). A Bayesian framework for simultaneously modeling neural and behavioral data. Neuroimage 72, 193-206. doi: 10.1016/j.neuroimage.2013.01.048

Turner, B. M., Rodriguez, C. A., Norcia, T. M., McClure, S. M., and Steyvers, M. (2016). Why more is better: simultaneous modeling of eeg, fMRI, and behavioral data. Neuroimage 128, 96-115. doi: 10.1016/j.neuroimage.2015. 12.030

van der Maaten, L., and Hinton, G. E. (2008). Visualizing data using t-SNE. J. Mach. Learn. Res. 9, 2579-2605.

Wardle, S. G., Kriegeskorte, N., Grootswagers, T., Khaligh-Razavi, S.-M., and Carlson, T. A. (2016). Perceptual similarity of visual patterns predicts dynamic neural activation patterns measured with MEG. Neuroimage 132, 59-70. doi: 10.1016/j.neuroimage.2016.02.019

Weinberg, A., Riesel, A., and Hajcak, G. (2012). Integrating multiple perspectives on error-related brain activity: the ERN as a neural indicator of trait 
defensive reactivity. Motiv. Emot. 36, 84-100. doi: 10.1007/s11031-0119269-y

Wiecki, T. V., and Frank, M. J. (2013). A computational model of inhibitory control in frontal cortex and basal ganglia. Psychol. Rev. 120, 329-355. doi: $10.1037 / \mathrm{a} 0031542$

Wiecki, T. V., Poland, J., and Frank, M. J. (2015). Model-based cognitive neuroscience approaches to computational psychiatry: clustering and classification. Clin. Psychol. Sci. 3, 378-399. doi: 10.1177/216770261 4565359

Wu, J., Quinlan, E. B., Dodakian, L., McKenzie, A., Kathuria, N., Zhou, R. J., et al. (2015). Connectivity measures are robust biomarkers of cortical function and plasticity after stroke. Brain 138, 2359-2369. doi: 10.1093/brain/ awv156

Wu, J., Srinivasan, R., Burke Quinlan, E., Solodkin, A., Small, S. L., and Cramer, S. C. (2016). Utility of EEG measures of brain function in patients with acute stroke. J. Neurophysiol. 115, 2399-2405. doi: 10.1152/jn.00978.2015

Wu, J., Srinivasan, R., Kaur, A., and Cramer, S. C. (2014). Resting-state cortical connectivity predicts motor skill acquisition. Neuroimage 91, 84-90. doi: 10.1016/j.neuroimage.2014.01.026
Yuan, F., and Luo, Z. (2012). "The EEG De-noising research based on wavelet and hilbert transform method," in Proceedings of the International Conference on Computer Science and Electronics Engineering (ICCSEE) (Hangzhou: IEEE), 361-365.

Zhang, H., Fell, J., Staresina, B. P., Weber, B., Elger, C. E., and Axmacher, N. (2015). $\gamma$ power reductions accompanys stimulus-specific representations of dynamic events. Curr. Biol. 25, 635-640. doi: 10.1016/j.cub.2015.01.011

Conflict of Interest Statement: The authors declare that the research was conducted in the absence of any commercial or financial relationships that could be construed as a potential conflict of interest.

Copyright (c) 2018 Bridwell, Cavanagh, Collins, Nunez, Srinivasan, Stober and Calhoun. This is an open-access article distributed under the terms of the Creative Commons Attribution License (CC BY). The use, distribution or reproduction in other forums is permitted, provided the original author(s) and the copyright owner are credited and that the original publication in this journal is cited, in accordance with accepted academic practice. No use, distribution or reproduction is permitted which does not comply with these terms. 